\title{
THE COST OF GETTING LOCAL MONOTONICITY
}

\author{
JOSEP FREIXAS AND SASCHA KURZ
}

\begin{abstract}
In [15] Manfred Holler introduced the Public Good index as a proposal to divide a public good among players. In its unnormalized version, i.e., the raw measure, it counts the number of times that a player belongs to a minimal winning coalition. Unlike the Banzhaf index, it does not count the remaining winning coalitions in which the player is crucial. Holler noticed that his index does not satisfy local monotonicity, a fact that can be seen either as a major drawback [9, $221 \mathrm{ff}$.$] or as an advantage [16.$

In this paper we consider a convex combination of the two indices and require the validity of local monotonicity. We prove that the cost of obtaining it is high, i.e., the achievable new indices satisfying local monotonicity are closer to the Banzhaf index than to the Public Good index. All these achievable new indices are more solidary than the Banzhaf index, which makes them as very suitable candidates to divide a public good.

As a generalization we consider convex combinations of either: the Shift index, the Public Good index, and the Banzhaf index, or alternatively: the Shift Deegan-Packel, Deegan-Packel, and Johnston indices.
\end{abstract}

Keywords: Public Good Index, local monotonicity, design of power indices, solidarity, individualism, fair division

MSC: 91A12, 91A80, 91B12

JEL: D72

\section{INTRODUCTION}

Consider a set of players who jointly make decisions under a given set of rules. Here we specialize to simple games and subclasses thereof. Power indices address the question of how much power collective decision rules, like a weighted (voting) rule, award to each individual player: is player $i$ more or less powerful than player $j$, and by how much? For an example of an applied voting power analysis in the EU, we refer the interested reader to e.g. [1, 6, 32].

Different power indices measure different aspects of power and there is still a lot of research in order to answer the question which index to choose, see e.g. 17. For a recent overview of different power indices see e.g. [5]. Many of these indices are based on decisiveness. A player is called decisive in a coalition if his/her deletion in the coalition changes its status from winning to losing, so that the individual is decisive or crucial for it. All power indices, the classical and the newly introduced ones, considered in this paper are indeed based on counting different types of decisiveness for players in coalitions.

Some particular rules, weighted games, specify that each player $i=1, \ldots, n$ has a specific voting weight $w_{i}$ and that a collective decision requires enough supporters such that their total weight equals or surpasses a decision quota $q$. Let $p_{i}$ be the power value assigned to player $i$ by a power index. The power index is called locally monotonic if $w_{i} \geq w_{j}$ implies $p_{i} \geq p_{j}$, i.e., a player $i$ who controls a large share of vote does not have less power than a player $j$ with smaller voting weight. Local 
monotonicity is considered as an essential requirement for power measures by many authors. Felsenthal and Machover [9, $221 \mathrm{ff}$.], for instance, argue that any a priori measure of power that violates local monotonicity, LM for brevity, is 'pathological' and should be disqualified as serving as a valid yardstick for measuring power. On the other hand e.g. in [16] it is argued that local non-monotonicity is a very valuable property of a power index, since it can reveal certain properties of the underlying decision rule that are overlooked otherwise.

Local monotonicity is an implication of the dominance postulate which is based on the desirability relation as proposed by Isbell [20]. This property formalizes that a player $i$ is at least as desirable as a player $j$ if for any coalition $S$, such that $j$ is not in $S$ and the union of $S$ and $\{j\}$ is a winning coalition, i.e., is able to pass the collective decision at hand, the union of $S$ and $\{i\}$ is also a winning coalition. A power index satisfies dominance if $p_{i} \geq p_{j}$ whenever $i$ dominates $j$.

Freixas and Gambarelli [10] use desirability to define reasonable power measures and note that the dominance postulate implies local monotonicity. In this paper we will consider the Public Good, the Banzhaf, the Shift, the Shift Deegan-Packel, the Deegan-Packel, the Johnston index and convex combinations thereof. Since the Deegan-Packel index [7, and the Public Good Index (see Holler [15; Holler and Packel [19]) violate local monotonicity, they also violate the dominance postulate. Moreover, any violation of local monotonicity for the Deegan-Packel index implies a violation of the Shift Deegan-Packel index (see [3]) and any violation of the local monotonicity for the Public Good Index implies a violation of the Shift index (see [2]). It is well-known that the Banzhaf [4] and Johnston [21] indices satisfy the dominance postulate and therefore local monotonicity. If one or several power indices violate LM then a convex combination with another power index, that does not violate LM, yields a power index that also does not violate LM as long as the weight of the latter index is large enough. To study how large this has to be is the purpose of this paper.

Some works are devoted to verify the properties of dominance or local monotonicity (among others) for some power indices and to show failures for some other power indices (see among others, Felsenthal and Machover [8] or Freixas et al. [12]). Other works are devoted to study subclasses of games for which a given power index not fulfilling local monotonicity satisfies it for such a subclass of games (see for instance, Holler et al. [18] and Holler and Napel [16] for the Public Good Index). Here we will also make a new contribution of this type, i.e., we consider two new subclasses of games for which the Public Good Index satisfies local monotonicity.

The strictest generalization of local monotonicity is proportionality of power and weights. For the classical power indices this property is satisfied for a subset of weighted games only. Power indices which generally satisfy this property are constructed in 22 .

This paper starts by modifying the Public Good index with the purpose to achieve a new power index being local monotonic and more solidary than the Banzhaf index. These two properties make those achievable power indices (if they exist) well-situated as yardstick for doing a fair division of a public good. The idea of such modification is nothing else than an hybrid between the original Public Good index and the Banzhaf index. It will turn out that the cost of obtaining local monotonicity is rather high, i.e., the achievable new indices satisfying local monotonicity are closer to the Banzhaf index than to the Public Good index. However 
these indices stress more in minimal winning coalitions, as the Public Good index does, than in the rest of crucial winning coalitions, with goes in the direction of Riker's size principle, see [31. The final result permits to find new indices being locally monotonic and being more solidary than the Banzhaf index, which makes them as good alternatives for the fair division of a public good among participants in the voting procedure.

The idea developed previously naturally extends when the raw Shift index is incorporated to the duo formed by the raw Public Good and raw Banzhaf indices. Local monotonic indices which are convex combinations of the three given raw indices are a further target of our research.

As an extension we do a similar study for convex combinations of the raw Johnston index, the raw Deegan-Packel index, and the raw Shift Deegan-Packel index.

The remaining part of the paper is organized as follows: In Section 2 we introduce the basic notation of games and power indices. Two subclasses of weighted games satisfying local monotonicity are presented in Section 3. The concept of considering convex combinations of some power indices as a new power index is outlined in Section 4 The cost of local monotonicity is introduced in the same section. Additionally we prove some structural results. An integer linear programming approach to compute the cost of local monotonicity is presented in Section 5 . With the aid of the underlying algorithm we are able to state some exact values and lower bounds for the cost of local monotonicity in Section 6. The set of all convex multipliers leading to a locally monotonic power index is the topic of Section 7. We end with a conclusion in Section 8 .

\section{NotAtion, GAMES AND INDiCES}

In the following we will denote the set of players, which jointly make a decision, by $N$ and assume w.l.o.g. that the players are numbered from 1 to $n$, i.e., $N=$ $\{1, \ldots, n\}$. Here we restrict ourselves to binary decisions, i.e., each player can either vote 1 , meaning 'yes', or 0 , meaning 'no', on a certain issue. We call a subset $S \subseteq N$, collecting the 'yes'-voters, coalition. A (binary) decision rule is formalized as a mapping $v: 2^{N} \rightarrow\{0,1\}$ from the set of possible coalitions to the set of possible aggregated decisions. It is quite natural to require that the aggregated decision transfers the players decision if they all coincide and that an enlarged set of supporters should not turn the decision from yes to no:

Definition 1. A simple game is a mapping $v: 2^{N} \rightarrow\{0,1\}$ such that $v(\emptyset)=0$, $v(N)=1$, and $v(S) \leq v(T)$ for all $S \subseteq T \subseteq N$.

Having local monotonicity in mind we additionally require that the players are linearly ordered according to their capabilities to influence the final group decision. This can be formalized, as already indicated in the introduction, with the desirability relation introduced in [20].

Definition 2. We write $i \sqsupset j$ (or $j \sqsubset i$ ) for two players $i, j \in N$ of a simple game $v$ if we have $v(\{i\} \cup S \backslash\{j\}) \geq v(S)$ for all $\{j\} \subseteq S \subseteq N \backslash\{i\}$ and we abbreviate $i \sqsupset j, j \sqsupset i$ by $i \square j$.

In words we say that $i$ dominates $j$ for $i \sqsupset j$ and we call $i$ and $j$ equivalent iff $i \square j$. 
Definition 3. A simple game $v$ is called complete if the binary relation $\sqsupset$ is a total preorder, i.e.,

(1) $i \sqsupset i$ for all $i \in N$,

(2) $i \sqsupset j$ or $j \sqsupset i$ for all $i, j \in N$, and

(3) $i \sqsupset j, j \sqsupset h$ implies $i \sqsupset h$ for all $i, j, h \in N$.

We call a coalition $S$ of a simple game $v$ winning if $v(S)=1$ and losing otherwise. Each simple game is uniquely characterized by its set $\mathcal{W}$ of winning coalitions (or its set $\mathcal{L}$ of losing coalitions). A winning coalition $S$ such that each of its proper subsets is losing is called a minimal winning coalition. The set $\mathcal{M}$ of minimal winning coalitions is already sufficient to uniquely characterize a simple game. For complete simple games the defining set of winning coalitions can be further reduced. A minimal winning coalition $S$ is called shift-minimal if for each pair of players $i$, $j$ with $i \in S, j \notin S, i \sqsupset j, j \not \supset i$ we have $v(S \backslash\{i\} \cup\{j\})=0$, i.e., replacing a player by a (properly) dominated player turns the coalition into a losing one. With this, each complete simple game is uniquely characterized by its set $\mathcal{S}$ of shift-minimal winning coalitions.

A very transparent form of dominance is induced by weights.

Definition 4. A simple game $v$ is called weighted (weighted game for brevity) if and only if there exist weights $w_{i} \in \mathbb{R}_{>0}$, for all $i \in N$, and a quota $q \in \mathbb{R}_{>0}$ such that $v(S)=1$ is equivalent to $w(S):=\sum_{i \in S} w_{i} \geq q$ for all $S \subseteq N$.

Given such a weighted representation we write $v=\left[q ; w_{1}, \ldots, w_{n}\right]$. All weighted games are complete. As remarked before $w_{i} \geq w_{j}$ implies that player $i$ dominates player $j$, i.e., $i \sqsupset j$, while $i \square j$ is still possible even for $w_{i}>w_{j}$.

In order to measure the influence of the players we use the concept of a power index, which we in general consider as a mapping from a set $\mathfrak{G}$ of games to a vector of $n$ real numbers, where $n$ is the number of players of the specific game. In most applications, considering subsets of the set of simple games, the image is a vector of $n$ non-negative real numbers upper bounded by 1 . To this end we denote by $\mathfrak{S}$ the set of simple games, by $\mathfrak{C}$ the set of complete simple games, and by $\mathfrak{W}$ the set of weighted games. In order to stress the underlying class of games, we speak of a power index $P$ on $\mathfrak{G}$, whenever it is not clear from the context.

In some contexts it is appropriate to further restrict the class of games:

Definition 5. A simple game is called proper if the complement $N \backslash S$ of any winning coalition $S$ is losing. It is called strong if the complement $N \backslash S$ of any losing coalition $S$ is winning. A simple game that is both proper and strong is called constant-sum (or self-dual, or decisive).

We will denote the restriction to proper, strong, or constant-sum games by a superscript p, s, and c, respectively, i.e., we write $\mathfrak{S}^{\mathrm{p}}, \mathfrak{S}^{\mathrm{s}}$, and $\mathfrak{S}^{\mathrm{c}}$ in the case of simple games. If $P$ is a power index on $\mathfrak{G}$, then there is a restricted power index $P^{\prime}$ on $\mathfrak{G}^{\prime}$ for all $\mathfrak{G}^{\prime} \subseteq \mathfrak{G}$,

Having the general concept of a power index $P$ on $\mathfrak{G}$ at hand, i.e., $P(v)=$ $\left(P_{1}(v), \ldots, P_{n}(v)\right)=\left(p_{1}, \ldots, p_{n}\right) \in \mathbb{R}^{n}$, we can define the properties that we are interested in this paper:

Definition 6. A power index $P$ on $\mathfrak{G} \subseteq \mathfrak{C}$ satisfies the dominance property if we have $p_{i} \geq p_{j}$ for all complete simple games $v \in \mathfrak{G}$ and all pairs of players $i \sqsupset j$, where $P(v)=\left(p_{1}, \ldots, p_{n}\right)$. 
Restricting the dominance property from the class of complete simple games to weighted games, we speak of local monotonicity.

Definition 7. A power index $P$ on $\mathfrak{G} \subseteq \mathfrak{W}$ satisfies local monotonicity (LM) if we have $p_{i} \geq p_{j}$ for all weighted games $v \in \mathfrak{G}$ and all pairs of players $i \sqsupset j$ (or $\left.w_{i} \geq w_{j}\right)$, where $P(v)=\left(p_{1}, \ldots, p_{n}\right)$.

We remark that the dominance property for the subclass of weighted games implies local monotonicity and local monotonicity implies symmetry, i.e., we have $p_{i}=p_{j}$ for all $i \square j$. Further properties of classical power indices where named and studied in the literature, e.g., if all $p_{i}$ 's are non-negative and sum up to one the power index is called efficient. If $p_{i}=0$ for all players $i$ not contained in any minimal winning coalition, also called null players or nulls, then the power index is said to satisfy the null player property. Removing a null player from a simple game (complete simple game or weighted game) $v$ results in a simple game (complete simple game or weighted game) $v^{\prime}$ - more formally $v^{\prime}:=2^{N \backslash\{i\}} \rightarrow\{0,1\}$ $v^{\prime}(S)=v(S)$ for all $S \subseteq N \backslash\{i\}$. If $p_{j}=p_{j}^{\prime}$ for all $j \in N \backslash\{i\}$ and $p_{i}=0$, where $P(v)=\left(p_{1}, \ldots, p_{n}\right)$ and $P\left(v^{\prime}\right)=\left(p_{1}^{\prime}, \ldots, p_{i-1}^{\prime}, p_{i+1}^{\prime}, \ldots, p_{n}^{\prime}\right)$, we call $P$ invariant for nulls. We call a power index strictly positive if $p_{i}>0$ for all non-null players $i$ and $p_{j}=0$ for all null players $j$.

In order to state the definition for the set of power indices mentioned in the introduction we call a winning coalition $S$ decisive for player $i \in S$ if $S \backslash\{i\}$ is losing. Given a simple game $v$, by $\mathcal{D}_{i}$ we denote the set of coalitions $\{i\} \subseteq S \subseteq N$ being decisive for player $i$. Similarly, we denote by $\mathcal{M}_{i}$ the set of minimal winning coalitions containing player $i$ and by $\mathcal{S}_{i}$ the set of shift-minimal winning coalitions containing player $i$ (provided that the game is complete). In order to specify a power index $P$ it suffices to define the mapping for each coordinate $P_{i}(v)=p_{i}$.

Definition 8. The (raw) Banzhaf index $\mathrm{Bz}^{r}$ and the (raw) Public Good index $\mathrm{PGI}^{r}$ of a simple game $v$ are given by $\mathrm{Bz}_{i}^{r}(v)=\left|\mathcal{D}_{i}\right|$ and $\mathrm{PGI}_{i}^{r}(v)=\left|\mathcal{M}_{i}\right|$. The (raw) Shift index of a complete simple game $v$ is given by $\mathrm{S}_{i}^{r}(v)=\left|\mathcal{S}_{i}\right|$.

The (raw) Banzhaf index $\mathrm{Bz}^{r}$, the (raw) Public Good index $\mathrm{PGI}^{r}$, and the (raw) Shift index $\mathrm{S}^{r}$ all are based on decisive coalitions since they count subsets of decisive coalitions containing a given player $i$. The Banzhaf index counts all decisive coalitions for each player, while the Public Good index counts only the minimal and the Shift index only the shift-minimal ones. Thus, for each player we have the inclusion

$$
\mathcal{S}_{i} \subseteq \mathcal{M}_{i} \subseteq \mathcal{D}_{i}
$$

Counting a certain subset of coalitions is the base for many power indices, see e.g. 27] for further examples. As defined several coalitions can be counted multiple times, i.e., a minimal winning coalition $S$ is counted for every player $i$ contained in $S$ in the computation of $\mathrm{PGI}^{r}$. If one wants to avoid this characteristic and instead count each distinguished coalition just once, then one can divide 'spoils' of each coalition equally among its decisive members. This general construction is called equal division version of a given power index (based on counting functions) in [27. Applying this construction on our first set of power indices we obtain the second set:

Definition 9. The (raw) Johnston index $\mathrm{Jo}^{r}$ and the (raw) Deegan-Packel index $\mathrm{DP}^{r}$ of a simple game $v$ are given by $\mathrm{Jo}_{i}^{r}(v)=\sum_{S \in \mathcal{D}_{i}} \frac{1}{\# \text { decisive players in } S}$ and 
$\operatorname{DP}_{i}^{r}(v)=\sum_{S \in \mathcal{M}_{i}} \frac{1}{|S|}$. The (raw) Shift Deegan-Packel index of a complete simple game $v$ is given by $\operatorname{SDP}_{i}^{r}(v)=\sum_{S \in \mathcal{S}_{i}} \frac{1}{|S|}$.

Note that in the two last definitions $|S|=$ \# decisive players in $S$, since all players in minimal winning (or in shift-minimal winning) coalitions are decisive in them.

\section{Two subclasses where the Public Good and the Deegan-Packel SATISFY LOCAL MONOTONICITY}

As it is well-known the the Banzhaf and the Johnston indices (and the ShapleyShubik too) satisfy both the dominance property and local monotonicity since all of them preserve the desirability relation (see e.g. [12]). The other power indices previously introduced in Section 2 and extensively analyzed in this paper do not preserve the desirability relation and consequently fail to fulfill both the dominance and the local monotonicity properties.

The purpose of this section is to provide subclasses of games, not introduced before, satisfying the dominance property or the local-monotonicity for the Public Good and the Deegan-Packel indices. As seen below the cost for one of these two power indices to satisfy the local monotonicity property for a given game is related to the proximity or remoteness of the game to be in these subclasses.

Definition 10. A simple game is:

(1) uniform if all minimal winning coalitions have the same cardinality,

(2) uniformly complete if it is both complete and uniform,

(3) uniformly weighted if it is both weighted and uniform.

As an abbreviation we denote the corresponding subclasses by $\mathfrak{U}, \mathfrak{U}_{c}$, and $\mathfrak{U}_{w}$, respectively. Obviously, a uniformly weighted game is a uniformly complete game and a uniformly complete game is a uniform game, while the converses are not true.

The second observation is that if a game is uniform then all shift-minimal winning coalitions have the same cardinality because $\mathcal{S} \subseteq \mathcal{M}$ for all simple game. However the converse is also true, i.e., if all shift-minimal winning coalitions have the same cardinality then all the minimal winning coalitions have the same cardinality too. This is because the coalitions in $\mathcal{M} \backslash \mathcal{S}$ are obtained from those in $\mathcal{S}$ by one-to-one replacements of weaker players, according to the desirability relation, for stronger players; but these exchanges do not affect the cardinalities of the coalitions involved. Hence, we can exchange in previous definition the term "minimal winning coalition" by "shift-minimal winning coalition".

If $l$ is the cardinality of all minimal winning coalitions in a uniform game, in any of its forms, then $\mathcal{M}=\mathcal{M}(l)$ where $\mathcal{M}(l)$ is the set of minimal winning coalitions of cardinality $l$.

Proposition 1. Let $v$ be a uniform complete simple game, characterized by $\mathcal{W}$ and $N$, then the Public Good and Deegan-Packel indices satisfy the dominance property.

Proof. Since $D P_{i}^{r}=P G I_{i}^{r} / l$ for all $i \in N$, where $l=\min \{|S|: S \in \mathcal{W}\}$, it suffices to prove the statement for the Public Good index.

Assume $i \sqsupset j$, then for all $S \subseteq N \backslash\{i, j\}$ with $S \cup\{j\} \in \mathcal{M}$ we have $S \cup\{i\} \in \mathcal{W}$. If $S \cup\{i\} \notin \mathcal{M}$, then there would exist a player $k \in S$ such that $(S \cup\{i\}) \backslash\{k\} \in \mathcal{W}$, but $|(S \cup\{i\}) \backslash\{k\}|=|S \cup\{j\}|-1=l-1$, which is a contradiction with the fact 
that all minimal winning coalitions have the same size. Thus, $S \cup\{i\} \in \mathcal{M}$ and therefore $\mathrm{PGI}_{i}^{r}=\left|\mathcal{M}_{i}\right| \geq\left|\mathcal{M}_{j}\right|=\mathrm{PGI}_{j}^{r}$.

We remark that $i \sqsupset j, i \not \supset j$ even implies $\mathrm{PGI}_{i}^{r}>\mathrm{PGI}_{j}^{r}$.

Corollary 1. The Public Good and Deegan-Packel indices satisfy dominance and local monotonicity properties on the classes $\mathfrak{U}_{c}$ and $\mathfrak{U}_{w}$, respectively.

As a consequence, for uniform complete simple games the ranking given by the desirability relation coincides with the rankings given by Public Good and DeeganPackel indices. So these ranking also coincide with the rankings of the ShapleyShubik, Banzhaf, and Johnston indices, see [12].

Despite the restrictive definition of uniform complete simple games, their number is large. For instance, there are (see e.g. 14, 29]) $2^{n}-1$ uniform complete simple games with just one type of shift-minimal winning coalitions of $n$ voters. Table 1 provides enumerations for small values of $n$ on the number of uniform complete games $\left(\mathfrak{U}_{c}\right)$ and uniform weighted games $\left(\mathfrak{U}_{w}\right)$. For $n=10$ players the number of uniform complete simple games is given by 3049712101 and for $n=11$ players the respective number larger than $25 \cdot 10^{12}$. Without considering symmetry the number of uniform simple games with $n$ players is given by $\sum_{k=1}^{n} 22^{\left(\begin{array}{l}n \\ k\end{array}\right)-1}$.

\begin{tabular}{|c|c|c|c|c|c|c|c|c|c|}
\hline \hline $\mathrm{n}$ & 1 & 2 & 3 & 4 & 5 & 6 & 7 & 8 & 9 \\
\hline \hline $\mathfrak{U}_{c}$ & 1 & 3 & 7 & 16 & 41 & 140 & 843 & 14434 & 1410973 \\
$\mathfrak{U}_{w}$ & 1 & 3 & 7 & 16 & 41 & 125 & 458 & 2188 & 20079 \\
\hline \hline
\end{tabular}

TABLE 1. Number of uniform complete simple games and uniform weighted games.

Being a uniform game is a sufficient condition for both the Public Good and the Deegan-Packel index to be local monotonic. However, this condition is not necessary as the following example illustrates.

Example 1. Let $v$ be the 4-person game uniquely characterized by $N=\{1,2,3,4\}$ and $\mathcal{M}=\{\{1,2\},\{1,3\},\{1,4\},\{2,3,4\}\}$, i.e., $v$ admits the weighted representation $[3 ; 2,1,1,1]$. This game is the unique weighted game of 4 voters which is not uniform but it satisfies the dominance property for the Public Good index.

At the very least, this game is captured by the following two definitions.

Definition 11. Let $v$ be a simple game with player set $N=\{1, \ldots, n\}$ and

$$
i \succsim j \quad \text { if and only if } \sum_{l=1}^{k}\left|\mathcal{M}_{i}(l)\right| \geq \sum_{l=1}^{k}\left|\mathcal{M}_{j}(l)\right| \text { for all } k=1,2, \ldots, n \text {. }
$$

Then $\succsim$ is a preordering on $N$, i.e., a reflexive and transitive relation, called the layer relation.

Definition 12. A simple game $v$ on $N$ is flat whenever $\succsim$, the layer relation on $N$, is total.

Thus, in a flat simple game, we either have $i \succsim j$ or $j \succsim i$ for all $i, j \in N$. Let $\mathfrak{F}$ be the class of flat games. 
Proposition 2. Let $v$ be a flat complete simple game then the Public Good and Deegan-Packel indices satisfy the dominance property.

Proof. Assume $i \sqsupset j$ and let $k \leq n-2$ be the maximal integer such that $\{S \subseteq N \backslash\{i, j\}: S \cup\{i\} \in \mathcal{M},|S|=k\}=\{S \subseteq N \backslash\{i, j\}: S \cup\{j\} \in \mathcal{M},|S|=k\}$. With this we have $\sum_{h=1}^{l} \mathcal{M}_{i}(h)=\sum_{h=1}^{l} \mathcal{M}_{j}(h)$ for all $l \leq k+1$. Now let $S \subseteq$ $N \backslash\{i, j\}$ be a coalition of cardinality $k+1$. If $S \cup\{j\} \in \mathcal{M}$, then $S \cup\{i\} \in \mathcal{W}$ and $S^{\prime} \cup\{j\} \notin \mathcal{M}$ for all $S^{\prime} \subsetneq S$. Due to the definition of $k$ we have $S^{\prime} \cup\{i\} \notin \mathcal{M}$ for all $S^{\prime} \subsetneq S$ and conclude $S \cup\{i\} \in \mathcal{M}$. Using the definition of $k$ again, we conclude $\sum_{h=1}^{k+2} \mathcal{M}_{i}(h)=\sum_{h=1}^{k+2} \mathcal{M}_{j}(h)$ or $k=m-2$, i.e., we must have $i \succsim j$.

Since $\mathrm{PGI}_{i}^{r}=\sum_{l=1}^{n}\left|\mathcal{M}_{i}(l)\right| \geq \sum_{l=1}^{n}\left|\mathcal{M}_{j}(l)\right|=\mathrm{PGI}_{j}^{r}$ the statement is true for the public Good index.

Since $\mathrm{DP}_{i}^{r}=\frac{1}{l} \sum_{l=1}^{n}\left|\mathcal{M}_{i}(l)\right|$ we have the decomposition

$$
\begin{aligned}
\mathrm{DP}_{i}^{r}-\mathrm{DP}_{j}^{r}= & \left(1-\frac{1}{2}\right)\left(\sum_{l=1}^{1}\left|\mathcal{M}_{i}(l)\right|-\sum_{l=1}^{1}\left|\mathcal{M}_{j}(l)\right|\right)+ \\
& \left(\frac{1}{2}-\frac{1}{3}\right)\left(\sum_{l=1}^{2}\left|\mathcal{M}_{i}(l)\right|-\sum_{h=1}^{2}\left|\mathcal{M}_{j}(l)\right|\right)+ \\
& \cdots+ \\
& \left(\frac{1}{n-1}-\frac{1}{n}\right)\left(\sum_{l=1}^{n-1}\left|\mathcal{M}_{i}(l)\right|-\sum_{h=1}^{n-1}\left|\mathcal{M}_{j}(l)\right|\right)+ \\
& \frac{1}{n}\left(\sum_{h=1}^{n}\left|\mathcal{M}_{i}(l)\right|-\sum_{h=1}^{n}\left|\mathcal{M}_{j}(l)\right|\right) .
\end{aligned}
$$

Since $v$ is flat, each addend is non-negative, i.e., $\mathrm{DP}_{i}^{r} \geq \mathrm{DP}_{j}^{r}$.

We remark that $i \sqsupset j, i \not \supset j$ even implies $\mathrm{DP}_{i}^{r}>\mathrm{DP}_{j}^{r}$. Note further that uniform games are particular cases of flat games.

Example 2. Let $v$ be the weighted game uniquely characterized by $N=\{1,2,3\}$ and $\mathcal{M}=\{\{1\},\{2,3\}\}$. This is a flat game with $1 \sqsupset 2,2 \not \supset 1$, and $2 \square 3$. The (normalized) Public Good index is given by $\frac{1}{3} \cdot(1,1,1)$, i.e., player 1 and player 2 obtain the same value while not being equivalent. The (normalized) Deegan-Packel index is given by $\frac{1}{4} \cdot(2,1,1)$.

Corollary 2. The Public Good and Deegan-Packel indices satisfy local monotonicity for weighted flat games.

\section{Convex COMbinations of POWER Indices And the COST OF LOCAL MONOTONICITY}

As mentioned in the introduction the aim of this paper is to study power indices arising as a convex combination of a given (finite) collection of power indices. To this end let $\mathcal{P}$ be a finite set of power indices, e.g. $\mathcal{P}=\left\{\mathrm{Bz}^{r}, \mathrm{PGI}^{r}\right\}$ or $\mathcal{P}=$ $\left\{\mathrm{Bz}^{r}, \mathrm{PGI}^{r}, \mathrm{~S}^{r}\right\}$, which we will use later on. Given a set $\mathcal{P}=\left\{P^{1}, \ldots, P^{r}\right\}$ of power indices we consider the convex combinations

$$
P^{\alpha, \mathcal{P}}=\sum_{i=1}^{r} \alpha_{i} \cdot P^{i}
$$

where $\alpha=\left(\alpha_{1}, \ldots, \alpha_{r}\right) \in[0,1]^{r}$ and $\sum_{i=1}^{r} \alpha_{i}=1$, that is $\alpha$ belongs to the $r$ dimensional simplex. For brevity we write $\alpha \in \mathbb{S}^{r}$. The power indices $P^{i}$ are defined on possibly different classes $\mathfrak{G}_{i}$ and we set $\mathfrak{G}=\cap_{i=1}^{r} \mathfrak{G}_{i}$. Obviously $P^{\alpha, \mathcal{P}}$ is a power index on $\mathfrak{G}$ too, i.e., it maps games in $\mathfrak{G}$ to a vector in $\mathbb{R}^{n}$. In the remaining 
part of the paper we will not explicitly mention the underlying classes $\mathfrak{G}_{i}$ and $\mathfrak{G}$ of games.

Convex combinations of power indices have the nice feature that they preserve the properties for power indices defined in Section 2

Lemma 1. Let $\mathcal{P}=\left\{P^{1}, \ldots, P^{r}\right\}$ be a collection of power indices such that $P^{j}$

(1) satisfies the null player property,

(2) is symmetric,

(3) is strictly positive,

(4) is efficient,

(5) has the dominance property,

(6) is local monotonic, or

(7) is invariant for nulls

for all $1 \leq j \leq r$, then $P^{\alpha, \mathcal{P}}$, where $\alpha \in \mathbb{S}^{r}$, has the same property.

Proof. For properties (1)-(3) the statement directly follows from the definition. For property (4) we have

$$
\sum_{i=1}^{n} P_{i}^{j}(v)=1
$$

for all $1 \leq j \leq r$. With this we conclude

$$
\sum_{i=1}^{n} P_{i}^{\alpha, \mathcal{P}}(v)=\sum_{i=1}^{n} \sum_{j=1}^{r} \alpha_{j} \cdot P_{i}^{j}(v)=\sum_{j=1}^{r} \alpha_{j} \cdot \sum_{i=1}^{n} P_{i}^{j}(v)=\sum_{j=1}^{r} \alpha_{j}=1,
$$

using the fact that the $\alpha_{j}$ sum up to one.

For properties (5) and (6) we consider players $i$ and $h$ such that $P_{i}^{j}(v) \geq P_{h}^{j}(v)$ for all $1 \leq j \leq r$. With this we have

$$
P_{i}^{\alpha, \mathcal{P}}(v)=\sum_{j=1}^{r} \alpha_{j} \cdot P_{i}^{j}(v) \geq \sum_{j=1}^{r} \alpha_{j} \cdot P_{h}^{j}(v)=P_{h}^{\alpha, \mathcal{P}}(v),
$$

since the $\alpha_{j}$ are non-negative.

If the $P^{j}$ are invariant for nulls, then they have to satisfy the null player property. From (1) we deduce that $P^{\alpha, \mathcal{P}}$ also satisfies the null player property. Thus it suffices to prove $P_{i}^{\alpha, \mathcal{P}}(v)=P_{i}^{\alpha, \mathcal{P}}\left(v^{\prime}\right)$ for every player $i$ and every game $v^{\prime}$ arising from $v$ by deleting an arbitrary null player. We have

$$
P_{i}^{\alpha, \mathcal{P}}(v)=\sum_{j=1}^{r} \alpha_{j} \cdot P_{i}^{j}(v)=\sum_{j=1}^{r} \alpha_{j} \cdot P_{i}^{j}\left(v^{\prime}\right)=P_{i}^{\alpha, \mathcal{P}}\left(v^{\prime}\right),
$$

so that the statement is also true for property (7).

In this paper we are especially interested in the case where not all but at least one power index of a collection $\left\{P^{1}, \ldots, P^{r}\right\}$ satisfies local monotonicity. W.l.o.g. we assume $r \geq 2$ and that $P^{1}$ satisfies local monotonicity while the other indices might or might not satisfy LM. The convex combinations $P^{\alpha, \mathcal{P}}$ may or may not satisfy LM, depending on $\alpha$. At least for $\alpha=(1,0, \ldots, 0)$ LM is satisfied.

Definition 13. Let $\mathfrak{G} \subseteq \mathfrak{W}$ be a class of weighted games, $n \in \mathbb{N}_{>0}$ and $P=$ $\left\{P^{1}, \ldots, P^{r}\right\}$ be a collection of power indices on $\mathfrak{G}$ such that $P^{1}$ satisfies local monotonicity. By $\mathbb{P}_{\mathrm{LM}}^{\mathcal{P}}(n, \mathfrak{G})$ we denote the set of $\alpha \in \mathbb{S}^{r}$ such that $P^{\alpha, \mathcal{P}}$ satisfies $L M$ on the set of games of $\mathfrak{G}$ consisting of $n$ players. 
Lemma 2. Given the requirements of Definition 13 , the set $\mathbb{P}_{\mathrm{LM}}^{\mathcal{P}}(n, \mathfrak{G})$ is a nonempty (bounded) polyhedron.

Proof. Obviously, we have $\mathbb{P}_{\mathrm{LM}}^{\mathcal{P}}(n, \mathfrak{G}) \subseteq \mathbb{S}^{r}$. Given a game $v \in \mathfrak{G}$ and two players $1 \leq i, j \leq n$ with $i \sqsupset j$, we have $P_{i}^{\alpha, \mathcal{P}}(v) \geq P_{j}^{\alpha, \mathcal{P}}(v)$ if and only if $P_{i}^{\alpha, \mathcal{P}}(v)-$ $P_{j}^{\alpha, \mathcal{P}}(v) \geq 0$, which is equivalent to

$$
\sum_{h=1}^{r} \alpha_{h} \cdot \underbrace{\left(P_{i}^{h}(v)-P_{j}^{h}(v)\right)}_{\in[0,1]} \geq 0
$$

Thus $\mathbb{P}_{\mathrm{LM}}^{\mathcal{P}}(n, \mathfrak{G})$ is given as the intersection of $\mathbb{S}^{r}$ and the half-spaces (and possibly $\mathbb{R}^{r}$ for trivial inequalities $0 \geq 0$ ) described by Inequality (2) for all $v \in \mathfrak{G}$ consisting of $n$ players and all $1 \leq i, j \leq n$ with $i \sqsupset j$. We have $\mathfrak{G} \subseteq \mathfrak{W} \subseteq \mathfrak{S}$, so that the number of $n$-player games of $\mathfrak{G}$ is upper bounded by the number of simple games consisting of $n$ players. Since each simple game is uniquely characterized by its set of winning coalitions the number of simple games with $n$ players is at most $2^{2^{n}}$, i.e., finite. Thus we have a finite intersection of half-spaces and a polyhedron, which is a, possibly empty, polyhedron. It remains to remark that $(1,0, \ldots, 0) \in \mathbb{P}_{\mathrm{LM}}^{\mathcal{P}}(n, \mathfrak{G})$ to conclude the non-emptiness.

Example 3. We consider $\mathcal{P}=\{\overbrace{\mathrm{Bz}^{r}}^{=: P^{1}}, \overbrace{\mathrm{PGI}^{r}}^{=: P^{2}}, \overbrace{\mathrm{S}^{r}}^{=: P^{3}}\}, \mathfrak{G}=\mathfrak{W}$, and $n=7$, i.e., the class of weighted games with 7 players. For $v=[2 ; 2,1,1,1,1,1,1]$ we have $\mathrm{Bz}^{r}(v)=$ $(7,5, \ldots, 5)$ and $\mathrm{PGI}^{r}(v)=\mathrm{S}^{r}(v)=(1,5, \ldots, 5)$. The corresponding inequality (2) for game $v$ and players 1,2 is given by $\alpha_{1} \cdot(7-5)+\alpha_{2} \cdot(1-5)+\alpha_{3} \cdot(1-5) \geq 0$. Inserting $\alpha_{2}+\alpha_{3}=1-\alpha_{1}$ yields $2 \alpha_{1}-4\left(1-\alpha_{1}\right) \geq 0$, which is equivalent to $\alpha_{1} \geq \frac{2}{3}$. Thus for all $\alpha$ with $\alpha_{1}<\frac{2}{3}$ the convex combination $P^{\alpha, \mathcal{P}}$ does not satisfy local monotonicity. This means that the weight of the Banzhaf index in a convex combination satisfying LM must be at least $\frac{2}{3}$, i.e., closer to the Banzhaf index than to the two other indices, on the set of weighted games with 7 players.

Example 4. Again we consider $\mathcal{P}=\{\overbrace{\mathrm{Bz}^{r}}^{=: P^{1}}, \overbrace{\mathrm{PGI}^{r}}^{=: P^{2}}, \overbrace{\mathrm{S}^{r}}^{=: P^{3}}\}, \mathfrak{G}=\mathfrak{W}$, and $n=7$. For $v=[14 ; 9,8,5,2,2,2,2]$ we have $\mathrm{Bz}^{r}(v)=(33,31,21,7,7,7,7), \operatorname{PGI}^{r}(v)=$ $(6,9,5,7,7,7,7)$, and $\mathrm{S}^{r}(v)=(1,8,5,4,4,4,4)$. The corresponding inequality (2) for the game $v$ and players 1,2 is given by $\alpha_{1} \cdot(33-31)+\alpha_{2} \cdot(6-9)+\alpha_{3} \cdot(1-8) \geq 0$. Inserting $\alpha_{1}=1-\alpha_{2}+\alpha_{3}$ yields $\alpha_{2} \leq \frac{2}{5}-\frac{9}{5} \cdot \alpha_{3}$ after a short calculation. Thus for all $\alpha$ with $\alpha_{2}>\frac{2}{5}-\frac{9}{5} \cdot \alpha_{3}$ the convex combination $P^{\alpha, \mathcal{P}}$ does not satisfy local monotonicity.

In Figure 1 we have depicted the two regions, where $P^{\alpha, \mathcal{P}}$ does not satisfy LM according to the weighted games of Example 3 and Example 4. The weight region, including its border, is a superset of $\mathbb{P}_{\mathrm{LM}}^{\mathcal{P}}(7, \mathfrak{W})$. Later on it will turn out it indeed coincides with $\mathbb{P}_{\mathrm{LM}}^{\mathcal{P}}(7, \mathfrak{W})$.

Focusing on the necessary impact of $P^{1}$ over the rest of the $P^{j}$ 's, we define the cost of local monotonicity $c_{\mathcal{P}}(n, \mathfrak{G})$ as the smallest value $\beta$ such that $P^{\alpha, \mathcal{P}}$ satisfies LM on $\mathfrak{G}$ for all $\alpha \in \mathbb{S}^{r}$ with $\alpha_{1} \geq \beta$. 


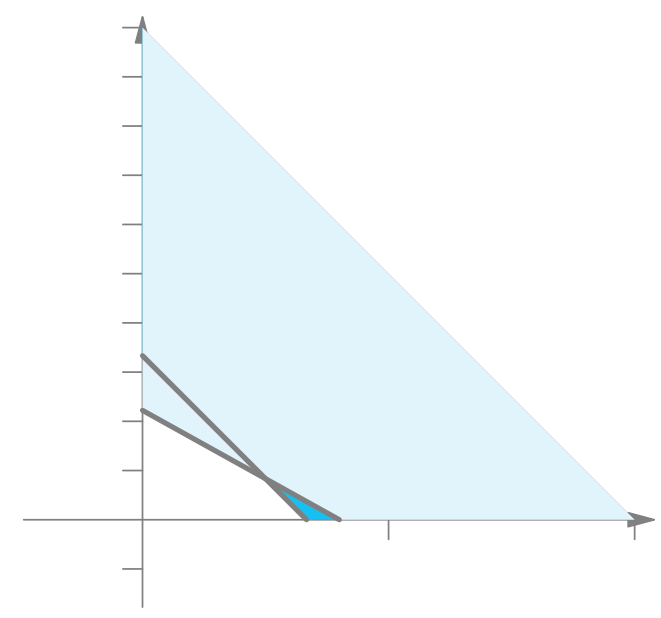

Figure 1. Regions of $P^{\alpha,\left\{\mathrm{Bz}^{r}, \mathrm{PGI}^{r}, \mathrm{~S}^{r}\right\}}$ which do not satisfy LM for weighted games with $n=7$ players.

Definition 14. Given the requirements of Definition 13, the cost of local monotonicity is defined as

$$
c_{\mathcal{P}}(n, \mathfrak{G})=\inf \left\{\beta \in[0,1] \mid P^{\alpha, \mathcal{P}} \text { satisfies LM on }(n, \mathfrak{G}), \quad \forall \alpha \in \mathbb{S}^{r}: \alpha_{1} \geq \beta\right\}
$$

for $r>1$, where $(n, \mathfrak{G})$ stands for the class of games $\mathfrak{G}$ with at most $n$ voters, and $c_{\mathcal{P}}(n, \mathfrak{G})=0$ for $r=1$.

Some examples may be derived from the previous section. For $\mathfrak{G}=\mathfrak{U}$, or more generally $\mathfrak{G}=\mathfrak{F}$, and $\mathcal{P}=\left\{J_{o}, D P\right\}$ we have $c_{\mathcal{P}}(n, \mathfrak{G})=0$ for all $n \in \mathbb{N}$, since the $D P$ index is local monotonic. Similarly, for $\mathfrak{G}=\mathfrak{U}$, or more generally $\mathfrak{G}=\mathfrak{F}$, and $\mathcal{P}=\{B z, P G I\}$ we have $c_{\mathcal{P}}(n, \mathfrak{G})=0$ for all $n \in \mathbb{N}$.

From Example 3 we conclude $c_{\left\{\mathrm{Bz}^{r}, \mathrm{PGI}^{r}, \mathrm{~S}^{r}\right\}}(7, \mathfrak{W}) \geq \frac{2}{3}$. The game from Example 4 gives the tighter inequality $c_{\left\{\mathrm{Bz}^{r}, \mathrm{PGI}^{r}, \mathrm{~S}^{r}\right\}}(7, \mathfrak{W}) \geq \frac{7}{9}$. Later on it will turn out that we can replace the infimum in Definition 14 by a minimum. Next we remark that dropping some of the power indices that do no satisfy LM does not increase the cost of local monotonicity:

Lemma 3. Given the requirements of Definition 13 , let $P^{1} \in \mathcal{P}^{\prime} \subseteq \mathcal{P}$ be a subset, then we have $c_{\mathcal{P}^{\prime}}(n, \mathfrak{G}) \leq c_{\mathcal{P}}(n, \mathfrak{G})$ for all $n \in \mathbb{N}_{>0}$.

Proof. For $\left|\mathcal{P}^{\prime}\right|=1$ the statement follows from $c_{\mathcal{P}}(n, \mathfrak{G}) \geq 0$. For $\left|\mathcal{P}^{\prime}\right|>1$ we can embed the elements of $\mathbb{S}^{\mid \mathcal{P}^{\prime}} \mid$ in $\mathbb{S}^{|\mathcal{P}|}$ by choosing zero for the missing indices.

Observation 1. Given the requirements of Definition 13 , we have $c_{\mathcal{P}}\left(n, \mathfrak{G}^{\prime}\right) \leq$ $c_{\mathcal{P}}(n, \mathfrak{G})$ for all $\mathfrak{G}^{\prime} \subseteq \mathfrak{G}$.

Under slight technical assumptions on the set of power indices and on $\mathfrak{G}$ we have monotonicity in the number of players:

Lemma 4. Given the requirements of Definition 13 , assume that all $P^{j}$ 's are invariant for nulls and that $\mathfrak{G}$ is closed under the addition of null players. Then we have $c_{\mathcal{P}}(n, \mathfrak{G}) \leq c_{\mathcal{P}}(n+1, \mathfrak{G})$ for all $n \in \mathbb{N}_{>0}$. 
Proof. Given an $n$-player game $v \in \mathfrak{G}$ with $P^{\alpha, \mathcal{P}}(v)=\left(p_{1}, \ldots, p_{n}\right)$, we can construct a game $v^{\prime} \in \mathfrak{G}$ by adding a null player such that $P^{\alpha, \mathcal{P}}\left(v^{\prime}\right)=\left(p_{1}, \ldots, p_{n}, 0\right)$. Since $P^{\alpha, \mathcal{P}}$ satisfies LM for $v$ if and only if $P^{\alpha, \mathcal{P}}$ satisfies LM for $v^{\prime}$, the statement follows.

For collections of $r=2$ power indices not only the set $\mathbb{P}_{\mathrm{LM}}^{\mathcal{P}}(n, \mathfrak{G})$ is a polyhedron but also its complement $\mathbb{S}^{r} \backslash \mathbb{P}_{\mathrm{LM}}^{\mathcal{P}}(n, \mathfrak{G})$ since both sets are intervals. Thus we can replace the infimum in Definition 14 by a minimum for all cases where $r=2$. Next we will show that the lower bounds from Lemma 3 for all subsets of cardinality two are sufficient to determine the cost of local monotonicity in general:

Lemma 5. Given the requirements of Definition 13, we have

$$
c_{\mathcal{P}}(n, \mathfrak{G})=\max \left\{c_{\left\{P^{1}, P^{j}\right\}}(n, \mathfrak{G}) \mid 2 \leq j \leq r\right\} .
$$

Proof. From Lemma 3 we conclude $c_{\mathcal{P}}(n, \mathfrak{G}) \geq \max \left\{c_{\left\{P^{1}, P^{j}\right\}}(n, \mathfrak{G}) \mid 2 \leq j \leq r\right\}$. W.l.o.g. we assume $c_{\left\{P^{1}, P^{j}\right\}}(n, \mathfrak{G})<1$ for all $2 \leq j \leq r$. Let $e_{i}$ denote the $i$ th unit vector. With this define $v_{j}=e_{1} \cdot c_{\left\{P^{1}, P^{j}\right\}}(n, \mathfrak{G})+e_{j} \cdot\left(1-c_{\left\{P^{1}, P^{j}\right\}}(n, \mathfrak{G})\right)$ for all $2 \leq j \leq r$. We have already observed that for $r=2$ the infimum in the definition of the cost of local monotonicity is indeed attained. Thus $P^{v_{j}, \mathcal{P}}$ satisfies LM. Since $\mathbb{P}_{\mathrm{LM}}^{\mathcal{P}}(n, \mathfrak{G}) \subseteq \mathbb{S}^{r}$ is convex, the $n-1$-dimensional $\operatorname{simplex} \operatorname{conv}\left(e_{1}, v_{2}, \ldots, v_{r}\right)=: \mathbb{F}$ is contained in $\mathbb{P}_{\text {LM }}^{\mathcal{P}}(n, \mathfrak{G})$. Now let $\mathbb{A}$ be the closure of $\mathbb{S}^{r} \backslash \mathbb{F}$, which is a polyhedron too. Indeed, the vertices of $\mathbb{A}$ are given by $v_{2}, \ldots, v_{r}$ and $e_{2}, \ldots, e_{r}$.

With this we have $c_{\mathcal{P}}(n, \mathfrak{G}) \leq \max \left\{a_{1} \mid\left(a_{1}, \ldots, a_{r}\right) \in \mathbb{A}\right\}$, where the maximum is attained at one of the $2 r-2$ vertices of $\mathbb{A}$. For the vertices $e_{2}, \ldots, e_{r}$ the respective $a_{1}$-value is zero. By definition the $a_{1}$-value of $v_{j}$ is given by $c_{\left\{P^{1}, P^{j}\right\}}$ for all $2 \leq j \leq$ $r$. Thus, $c_{\mathcal{P}}(n, \mathfrak{G}) \leq \max \left\{c_{\left\{P^{1}, P^{j}\right\}}(n, \mathfrak{G}) \mid 2 \leq j \leq r\right\}$.

\section{Corollary 3.}

$$
c_{\mathcal{P}}(n, \mathfrak{G})=\min \left\{\beta \in \mathbb{R}_{\geq 0} \mid P^{\alpha, \mathcal{P}} \text { satisfies } L M \text { on } \mathfrak{G} \forall \alpha \in \mathbb{S}^{r}: \alpha_{1} \geq \beta\right\}
$$

So, in order to determine $c_{\mathcal{P}}(n, \mathfrak{G})$ it suffices to determine $c_{\left\{P^{1}, P^{h}\right\}}(n, \mathfrak{G})$ for all $2 \leq h \leq r$. Given a game $v \in \mathfrak{G}$ and two players $i, j \in N$ with $i \sqsupset j$, we can solve Inequality (2) for $\alpha_{1}$ using $\alpha_{1}+\alpha_{h}=1$ If $P^{h}$ violates LM for $v$ and players $i, j$, we obtain an inequality of the form $\alpha_{1} \geq u$ and can conclude $c_{\left\{P^{1}, P^{h}\right\}}(n, \mathfrak{G}) \geq u$. ¿From Example 3 we conclude $c_{\left(\mathrm{Bz}^{r}, \mathrm{PGI}^{r}\right)}(7, \mathfrak{W}) \geq \frac{2}{3}$ and from Example 4 we conclude $c_{\left(\mathrm{Bz}^{r}, \mathrm{PGI}^{r}\right)}(7, \mathfrak{W}) \geq \frac{7}{9}$.

In order to obtain tight bounds for the cost of local monotonicity, we may simply loop over all possible choices of $v, i$, and $j$. At the very least, we can partially restrict the number of choices as follows: Assuming $1 \sqsupset \cdots \sqsupset n$ the requirement $i \sqsupset j$ is equivalent to $i \leq j$. We remark that if $P^{h}$ violates $\operatorname{LM}$ for $v$ and players $i, j$ and there exists another player $i<i^{\prime}<j$, then LM is violated for $v$ and at least one of the pairs $i, i^{\prime}$ or $i^{\prime}, j$ of players. Thus, we can restrict our considerations on pairs of players of the form $i, i+1$, where $1 \leq i<n$.

Lemma 6. Given the requirements of Definition 13 with $r=2$, we have

$$
c_{\left(P^{1}, P^{2}\right)}(n, \mathfrak{G})=\max \left\{l_{i}(v) \mid v \in \mathfrak{G}, 1 \leq i \leq n-1\right\},
$$

where

$$
\begin{aligned}
& l_{i}(v):=\left(P_{i}^{2}(v)-P_{i+1}^{2}(v)\right) /\left(P_{i+1}^{1}(v)-P_{i}^{1}(v)+P_{i}^{2}(v)-P_{i+1}^{2}(v)\right) \\
& \text { if } P_{i+1}^{1}(v)-P_{i}^{1}(v)+P_{i}^{2}(v)-P_{i+1}^{2}(v)>0 \text { and } l_{i}(v):=0 \text { otherwise. }
\end{aligned}
$$


The big drawback of this exact approach is the usually large size of the set of $n$ player games of $\mathfrak{G}$. Both sets of $n$-player complete simple or weighted games grow faster than exponential. The exact numbers have been determined up to $n=9$ only, see e.g. 24] for the numbers of complete and weighted games up to $n=8$, 13] for the number of complete games for $n=9$, and [25, 29] for the number of weighted games for $n=9$. For $n=9$ there are 284432730174 complete simple and 993061482 weighted games 11 Thus, using Lemma 6 becomes computationally infeasible for $n>9$. So we propose an integer linear programming formulation in the next section.

\section{AN INTEGER LINEAR PROGRAMMING FORMULATION}

Whenever one is interested in complete simple games or weighted games, which are extremal with respect to a certain criterion, exhaustive enumeration is not a feasible option for $n>9$ players, see the enumeration results stated at the end of the previous section. An alternative is to specify the set games indirectly by binary variables and linear inequalities. If the extremality criterion can be also formulated using integer variables and linear constraints, then integer linear programming techniques can be applied. In the context of cooperative games this approach was introduced in [26] and also applied in this context in e.g. [11, 27, 28].

For completeness, we briefly repeat the ILP formulation of a game $v$. Since $v$ is uniquely characterized by its values $v(S)$ for all coalitions $S \in 2^{N}$, we introduce binary variables $x_{S} \in\{0,1\}$ for all $S \in 2^{N}$. The conditions for a simple game can be stated as $x_{\emptyset}=0, x_{N}=1$, and $x_{S} \leq x_{T}$ for all $S \subseteq T \subseteq N$. We remark that for the later set of inequalities it suffices to consider the pairs of coalitions where $|T|=|S|+1$. Complete simple games can be modeled by additionally requiring $x_{S} \leq x_{T}$ for all pairs of coalitions with $t_{i} \sqsupset s_{i}$ for $1 \leq i \leq m$, where $S=\left\{s_{1}, \ldots, s_{m}\right\}$ and $T=\left\{t_{1}, \ldots, t_{m}\right\}$.

In order to restrict $v$ to weighted games we additionally have to introduce weights $w_{i} \geq 0$ and a quota $q>0$, where we assume that the weight of each winning coalition is larger than the weight of each losing coalition by at least one. (We may simply use integer weights, which could result in harder problems for the ILP solver.) To interlink the $x_{S}$ with the $w_{i}$ and $q$ we use

$$
\begin{array}{r}
q-\left(1-x_{S}\right) \cdot M-\sum_{i \in S} w_{i} \leq 0 \quad \forall S \in 2^{N} \text { and } \\
-x_{S} \cdot M+\sum_{i \in S} w_{i} \leq q-1 \quad \forall S \in 2^{N},
\end{array}
$$

where $M$ is a suitably large constant fulfilling $M-1 \geq \sum_{i=1}^{n} w_{i}$. (We may choose $M=4 n\left(\frac{n+1}{4}\right)^{(n+1) / 2}$, see [30, Theorem 9.3.2.1].)

The restrictions to proper games can be formulated via $x_{S}+x_{N \backslash S} \leq 1$ for all $S \subseteq N$ with $|S| \leq \frac{n}{2}$. Similarly we can restrict to strong games by requesting $x_{S}+x_{N \backslash S} \geq 1$. For constant sum games we need $x_{S}+x_{N \backslash S}=1$.

In order to compute the power distribution of $\mathrm{Bz}^{r}, \mathrm{PGI}^{r}$, and $\mathrm{S}^{r}$ from the $x_{S}$, we introduce further binary variables, cf. [27]. For $i \in N$ and $S \in 2^{N}$ we set $y_{i, S}=1$ if and only if coalition $S$ is a swing for player $i$ and $y_{i, S}=0$ otherwise. This can

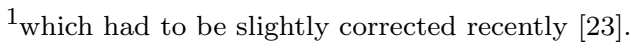


be ensured by requesting $y_{i, S}=0$ for $i \notin S$ and $y_{i, S}=x_{S}-x_{S \backslash\{i\}}$ otherwise. Similarly we introduce $z_{S} \in\{0,1\}$, where $z_{S}=1$ if and only if $S$ is a minimal winning coalition. This condition can be linearly reformulated as

$$
\begin{array}{rlrl}
z_{S}-x_{S} & \leq 0 & & \forall S \in 2^{N}, \\
z_{S}+x_{S \backslash\{i\}} \leq 1 & & \forall S \in 2^{N}, i \in S, \text { and } \\
z_{S}-x_{S}+\sum_{i \in S} x_{S \backslash\{i\}} & \geq 0 & & \forall S \in 2^{N} .
\end{array}
$$

In order to identify shift-minimal winning coalitions, we introduce binary variables $u_{S} \in\{0,1\}$ for all $S \in 2^{N}$. In order to state characterizing linear constraints we additionally need binary variables $t_{i} \in\{0,1\}$ for all $1 \leq i \leq n-1$, which are equal to 0 if and only if players $i$ and $i+1$ are of the same type, i.e., $i \square i+1$. This equivalence can be ensured by requesting

$$
\begin{aligned}
x_{S \cup\{i\}}-x_{S \cup\{i+1\}}-t_{i} & \leq 0 \quad \forall 1 \leq i \leq n-1, S \subseteq N \backslash\{i, i+1\} \text { and } \\
-t_{i}+\sum_{S \subseteq N \backslash\{i, i+1\}} x_{S \cup\{i\}}-x_{S \cup\{i+1\}} & \geq 0 \quad \forall 1 \leq i \leq n-1 .
\end{aligned}
$$

If $t_{i}=0$, i.e., $i \square i+1$, then $S \cup\{i\}$ is a shift-minimal winning coalition if and only if $S \cup\{i+1\}$ is a shift-minimal winning coalition, where $S \subseteq N \backslash\{i, i+1\}$ and $1 \leq i \leq n-1$. This conditioned equivalence can be expressed as

$$
u_{S \cup\{i\}} \geq u_{S \cup\{i+1\}}-t_{i} \text { and } u_{S \cup\{i+1\}} \geq u_{S \cup\{i\}}-t_{i} .
$$

So in the following we can restrict our considerations on coalitions $S$ such that for each $i \in S$ we either have $i=n, i+1 \in S$ or $i \sqsupset i+1$.

Since each shift-minimal winning coalition has to be a minimal winning coalition, we require $u_{S} \leq z_{S}$ for all $S \in 2^{N}$. The other possibility disqualifying a coalition $S$ from being a shift-minimal winning coalition is the existence of player $i \in S \backslash\{n\}$ with $i+1 \notin S$ and $i \sqsupset i+1$ such that $S \cup\{i+1\} \backslash\{i\}$ is winning. So we require

$$
u_{S} \leq 1+x_{S}-x_{S \cup\{i+1\} \backslash\{i\}}-t_{i}
$$

for all $S \subseteq N, n \neq i \in S$ and $i+1 \notin S$. Since $x_{S} \geq x_{S \cup\{i+1\} \backslash\{i\}}$ the right hand side is at least zero. So let us assume $t_{i}=1$. Since $u_{S} \leq z_{S} \leq x_{S}$ it suffices to consider the cases where $x_{S}=1$. If $x_{S \cup\{i+1\} \backslash\{i\}}=1$ then the stated inequality is trivially true. Just in the single case, where $x_{S}=1, x_{S \cup\{i+1\} \backslash\{i\}}=0$, and $t_{i}=1$, it implies $u_{S}=0$.

By now we can guarantee that $u_{S}=0$ if $S$ is not a shift-minimal winning coalition. However $u_{S}=0$ is still feasible for shift-minimal winning coalitions. So, we additionally require

$$
u_{S}-x_{S}+\sum_{i \in N \backslash\{n\}: i \in S, i+1 \notin S} x_{S \cup\{i+1\} \backslash\{i\}} \geq 0
$$

for all $S \subseteq N \backslash n$ and

$$
u_{S}-x_{S}+x_{S \backslash\{n\}}+\sum_{i \in N \backslash\{n\}: i \in S, i+1 \notin S} x_{S \cup\{i+1\} \backslash\{i\}} \geq 0
$$

for all $\{n\} \subseteq S \subseteq N$. If $x_{S}=0$ or one of the $x_{S \cup\{i+1\} \backslash\{i\}}=1$, then the proposed inequality is trivially satisfied. So we assume otherwise. In the cases where $n \in S$ we can similarly assume $x_{S \backslash\{n\}}=0$. In this remaining case we have the implication 
$u_{S}=1$, which is correct since no certificate for not being a shift-minimal winning coalition exists, so that $S$ is a shift-minimal winning coalition.

Finally consider the case where $S$ indeed is a shift-minimal winning coalition. Thus $x_{S}=1$. Remember that we need the implication $u_{S} \geq 1$ just for the coalitions $S$, where for each player $i \in S$ we either have $i=n, i+1 \in S$, or $i \sqsupset i+1$. So we can assume $i \sqsupset i+1$ for all indices $i$ in the summation. Thus $x_{S \cup\{i+1\} \backslash\{i\}}=0$. If $n \in S$, then also $x_{S \backslash\{n\}}=0$ since $S$ is a minimal winning coalition.

Having these variables at hand, we can easily compute the following power indices:

$$
\begin{aligned}
\mathrm{Bz}_{i}^{r}(v) & =\sum_{S \subseteq N} y_{i, S}, \\
\operatorname{PGI}_{i}^{r}(v) & =\sum_{\{i\} \subseteq S \subseteq N} z_{S}, \\
\mathrm{~S}_{i}^{r}(v) & =\sum_{\{i\} \subseteq S \subseteq N} u_{S}, \\
\operatorname{DP}_{i}^{r}(v) & =\sum_{\{i\} \subseteq S \subseteq N} \frac{1}{|S|} \cdot z_{S}, \text { and } \\
\operatorname{SDP}_{i}^{r}(v) & =\sum_{\{i\} \subseteq S \subseteq N} \frac{1}{|S|} \cdot u_{S} .
\end{aligned}
$$

For the Johnston index we have to take care that only the swing players obtain an equal share for each coalition. To this end we introduce the continuous variables $b_{i, S} \in \mathbb{R}_{\geq 0}$ for all $i \in N$ and and all $S \in 2^{N}$, cf. [27]:

$$
\begin{aligned}
b_{i, S} & \leq y_{i, S} \quad \forall S \in 2^{N}, i \in N \\
b_{i, S}-b_{j_{S}} & \geq y_{i, S}+y_{j, S}-2 \quad \forall S \in 2^{N}, i, j \in N \\
\sum_{i=1}^{n} b_{i, S} & \leq 1 \quad \forall S \in 2^{N} \\
\sum_{j=1}^{n} b_{i, S} & \geq y_{i, S} \quad \forall S \in 2^{N}, i \in N .
\end{aligned}
$$

Given an arbitrary coalition $S \subseteq N$, we can easily check that $\sum_{i=1}^{n} b_{i, S}=0$ if $S$ is not a swing coalition for any player $i \in N$ and $\sum_{i=1}^{n} b_{i, S}=1$ otherwise. In the later case we have $b_{i, S}=0$ whenever player $i$ is not a swing for coalition $S$. The second set of inequalities guarantees $b_{i, S}=b_{j, S}$ whenever both $i$ and $j$ are swings for coalition $S$. So we can state

$$
\mathrm{Jo}_{i}^{r}(v)=\sum_{S \subseteq N} b_{i, S}
$$

As a target we maximize

$$
P_{i+1}^{\alpha, \mathcal{P}}(v)-P_{i}^{\alpha, \mathcal{P}}(v)=\sum_{h=1}^{r} \alpha_{h} P_{i+1}^{h}(v)-\alpha_{h} P_{i}^{h}(v),
$$


where $1 \leq i \leq n-1$ has to be specified as a parameter. By looping over all possible values of $i$ we can decide whether $P^{\alpha, \mathcal{P}}$ satisfies LM for a given $\alpha \in \mathbb{S}^{r}$.

Lemma 7. For $\mathcal{P} \subseteq\left\{\mathrm{Bz}^{r}, \mathrm{PGI}^{r}, \mathrm{~S}^{r}, \mathrm{Jo}^{r}, \mathrm{DP}^{r}, \mathrm{SDP}^{r}\right\}, r:=|\mathcal{P}|$, and $\alpha \in \mathbb{S}^{r}$, one can decide $\alpha \in \mathbb{P}_{L M}^{\mathcal{P}}(n, \mathfrak{G})$ if incidence vectors of the $n$-player games in $\mathfrak{G}$ form a polyhedron.

To be more precise, we have explicitly stated ILP formulations for the classes of games $\mathfrak{G} \in\left\{\mathfrak{S}, \mathfrak{C}, \mathfrak{W}, \mathfrak{S}^{p}, \mathfrak{C}^{p}, \mathfrak{W}^{p}, \mathfrak{S}^{s}, \mathfrak{C}^{s}, \mathfrak{W}^{s}, \mathfrak{S}^{c}, \mathfrak{C}^{c}, \mathfrak{W}^{c}\right\}$. Having the binary variables $t_{i}$ at hand restrictions on the number of equivalence classes of players or even the precise partition can be formulated easily. ILP formulations for further power indices can be found in [27].

We assume that the algorithm behind Lemma 7 gives either the answer yes, if all corresponding $n-1$ ILPs have an optimal target value of zero, or gives the answer no together with a game $v \in \mathfrak{G}$ and an index $1 \leq i \leq n-1$ such that $P^{\alpha, \mathcal{P}}$ violates LM for the game $v$ and players $i, i+1$. The following algorithm computes the cost of local monotonicity for convex combinations of two power indices, i.e., $\mathcal{P}=\left\{P^{1}, P^{h}\right\}$ :

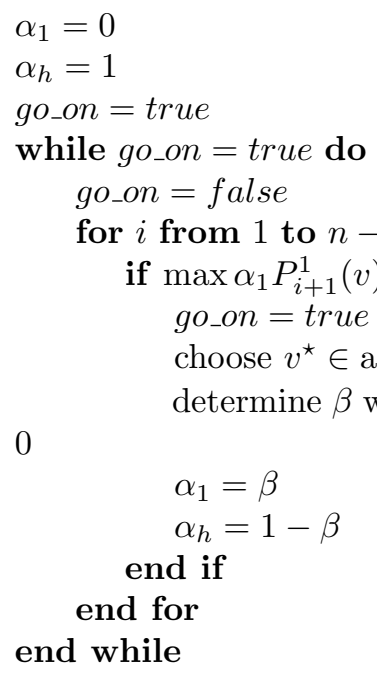

6. Exact VAlues AND LOWER BOUNDS FOR THE COST OF LOCAL MONOTONICITY

By considering parametric examples we can obtain general lower bounds for the cost of local monotonicity.

Lemma 8. For $\mathcal{P}=\left(\mathrm{Bz}^{r}, \mathrm{PGI}^{r}\right)$ and $n \geq 2$ we have $c_{\mathcal{P}}(n, \mathfrak{W}) \geq \max \left(0, \frac{n-3}{n-1}\right)$.

Proof. Since $c_{\mathcal{P}}(n, \mathfrak{W}) \geq 0$ by definition, it suffices to consider weighted games with $n \geq 4$ players. For the weighted game $v=[2 ; 2,1, \ldots, 1]$, with $n-1$ players of weight 1 and one player of weight 2 , the minimal winning coalitions are given by $\{1\}$ and $\{i, j\}$, where $2 \leq i<j \leq n$. Thus, we have $\operatorname{PGI}_{1}^{r}(v)=1$ and $\operatorname{PGI}_{2}^{r}(v)=n-2$. For player 1 the swing coalitions are given by $\{1\}$ and $\{1, i\}$ for all $2 \leq i \leq n$. Given a player $j \geq 2$ the swing coalitions for player $j$ are given by $\{i, j\}$ for all $2 \leq i \leq n$, with $i \neq j$. Thus, we have $\mathrm{Bz}_{1}^{r}(v)=n$ and $\mathrm{Bz}_{2}^{r}(v)=n-2$. 
For players 1, 2 and game $v$ Inequality (2) reads

$$
\alpha_{1} \cdot \mathrm{Bz}_{1}^{r}(v)+\alpha_{2} \cdot \operatorname{PGI}_{1}^{r}(v) \geq \alpha_{1} \cdot \operatorname{Bz}_{2}^{r}(v)+\alpha_{2} \cdot \operatorname{PGI}_{2}^{r}(v),
$$

which is equivalent to

$$
\alpha_{1} \cdot n+\alpha_{2} \cdot 1 \geq \alpha_{1} \cdot(n-2)+\alpha_{2} \cdot(n-2) \quad \Longleftrightarrow \quad \alpha_{1} \geq \frac{n-3}{n-1},
$$

since $\alpha_{1}+\alpha_{2}=1$.

Corollary 4. For $\mathcal{P}=\left(\mathrm{Bz}^{r}, \mathrm{~S}^{r}\right)$ and $n \geq 2$ we have $c_{\mathcal{P}}(n, \mathfrak{W}) \geq \max \left(0, \frac{n-3}{n-1}\right)$.

Proof. Since all minimal winning coalitions in the example of the proof of Lemma 8 are also shift-minimal winning, we can apply the same proof for the Shift index.

So, from Lemma 8, Corollary 4 , and Lemma 5 we can conclude that the cost of local monotonicity is at least $\frac{n-3}{n-1}$ for $\mathcal{P}=\left(\mathrm{Bz}^{r}, \mathrm{PGI}^{r}, \mathrm{~S}^{r}\right)$ and all $n \geq 2$.

\section{Corollary 5.}

$$
\lim _{n \rightarrow \infty} c_{\left(\mathrm{Bz}^{r}, \mathrm{PGI}^{r}\right)}(n, \mathfrak{W})=\lim _{n \rightarrow \infty} c_{\left(\mathrm{Bz}^{r}, \mathrm{~S}^{r}\right)}(n, \mathfrak{W})=\lim _{n \rightarrow \infty} c_{\left(\mathrm{Bz}^{r}, \mathrm{PGI}^{r}, \mathrm{~S}^{r}\right)}(n, \mathfrak{W})=1
$$

In other words, the only convex combination of $\mathrm{Bz}^{r}, \mathrm{PGI}^{r}$, and $\mathrm{S}^{r}$ that is locally monotonic for all weighted games is the raw Banzhaf index itself. For a finite number of players it may still be possible that is cost of local monotonicity is strictly less than 1.

Having the ILP approach from the previous section at hand we can also determine the exact value of the cost of local monotonicity for a small number of players. It turns out that the lower bound from Lemma 8 is tight for all $n \leq 10$. So, especially for $n \leq 3$ we have a cost of local monotonicity of zero, which goes in line with the fact that all weighted games with at most 3 players are locally monotonic. Although the ILP approach can move the computational limit of exhaustive enumeration a bit, it is so far limited to $n \leq 10$. Since we were not able to find worser examples, we state:

Conjecture 1. For $\mathcal{P}=\left(\mathrm{Bz}^{r}, \mathrm{PGI}^{r}\right)$ and $n \geq 2$ we have $c_{\mathcal{P}}(n, \mathfrak{W})=\max \left(0, \frac{n-3}{n-1}\right)$.

We remark that the extremal examples are not unique, e.g., we have the weighted games $[4 ; 4,3,2,2,1,1],[10 ; 10,8,5,4,4,3,3,2]$ and $[7 ; 7,6,6,6,5,3,3,3,2,1]$ also meeting the bound from Lemma 8 for $n=6, n=8$, and $n=10$, respectively. The respective Banzhaf scores are given by $(11,9,5,5,3,3),(28,26,16,12,12,10,10,6)$, and $(24,22,22,22,20,12,12,12,8,6)$. The respective PGI scores are given by $(1,4,3,3,3,3)$, $(1,6,11,9,9,8,8,6)$, and $(1,8,8,8,7,9,9,9,7,6)$.

For combinations of the Banzhaf score and the Shift score the lower bound of Corollary 4 is tight for $n \leq 6$. For $7 \leq n \leq 11$ we were able to computationally find worser examples.

Lemma 9. Let $\mathcal{P}=\left(\mathrm{Bz}^{r}, \mathrm{~S}^{r}\right)$.

(1) For $n=7$ we have $c_{\mathcal{P}}(n, \mathfrak{W})=\frac{7}{9} \approx 0.77777$.

(2) For $n=8$ we have $c_{\mathcal{P}}(n, \mathfrak{W})=\frac{7}{8}=0.875$.

(3) For $n=9$ we have $c_{\mathcal{P}}(n, \mathfrak{W})=\frac{25}{27} \approx 0.92593$.

(4) For $n=10$ we have $c_{\mathcal{P}}(n, \mathfrak{W})=\frac{51}{53} \approx 0.96226$.

(5) For $n=11$ we have $c_{\mathcal{P}}(n, \mathfrak{W})=\frac{97}{99} \approx 0.97980$. 
(6) For $n=12$ we have $c_{\mathcal{P}}(n, \mathfrak{W}) \geq \frac{175}{177} \approx 0.98870$.

PROOF. For the lower bounds we state an explicit weighted game and the Banzhaf and the Shift score for the first two players:

(1) $[14 ; 9,8,5,2,2,2,2], \mathrm{Bz}^{r}=(33,31, \ldots), \mathrm{S}^{r}=(1,8, \ldots)$;

(2) $[16 ; 11,10,5,2,2,2,2,2], \mathrm{Bz}^{r}=(65,63, \ldots), \mathrm{S}^{r}=(1,15, \ldots)$;

(3) $[30 ; 16,15,7,7,3,3,3,3,3], \mathrm{Bz}^{r}=(129,127, \ldots), \mathrm{S}^{r}=(2,27, \ldots)$, $[18 ; 13,12,5,2,2,2,2,2,2], \mathrm{Bz}^{r}=(129,127, \ldots), \mathrm{S}^{r}=(1,26, \ldots)$;

(4) $[33 ; 19,18,7,7,3,3,3,3,3,3], \mathrm{Bz}^{r}=(257,255, \ldots), \mathrm{S}^{r}=(2,53, \ldots)$;

(5) $[36 ; 22,21,7,7,3,3,3,3,3,3,3], \mathrm{Bz}^{r}=(513,511, \ldots), \mathrm{S}^{r}=(2,99, \ldots)$.

(6) $[56 ; 29,28,9,9,9,4,4,4,4,4,4,4], \mathrm{Bz}^{r}=(1025,1023, \ldots), \mathrm{S}^{r}=(2,177, \ldots)$.

For the upper bounds we have applied the ILP approach.

In some cases we have already stated different tight examples. We would highlight just another tight example, where the bound is met between the second and the third player, for $n=9$ : $[8 ; 5,3,2,2,2,2,2,2,2]$ with Banzhaf score $(85,43,41,41,41,41,41,41,41)$ and Shift score $(22,1,26,26,26,26,26,26,26)$.

We provide a general construction meeting the best known examples for all $n \geq 6$ :

Lemma 10. Let $k \geq 1$ be an integer, $m \in\{0,1,2\}, n=3 k+3+m$, and

$v=[2 t+m(k+1) ; t+1+m(k+1), t+m(k+1), \overbrace{2 k+3, \ldots, 2 k+3}^{k \text { times }}, \overbrace{k+1, \ldots, k+1}^{2 k+1+m \text { times }}$, where $t=2 k^{2}+3 k+1$. With this, $v$ is a weighted game consisting of $n$ players, $\mathrm{Bz}_{1}^{r}(v)=2^{n-2}+1, \mathrm{Bz}_{2}^{r}(v)=2^{n-2}-1, \mathrm{~S}_{1}^{r}(v)=1$ for $k=1, \mathrm{~S}_{1}^{r}(v)=2$ for $k>1$, and

$$
\mathrm{S}_{2}^{r}(v)=-1+\mathrm{S}_{1}^{r}(v)+\sum_{a=0}^{k} \sum_{b=\left\lceil\frac{t-a(2 k+3)}{k+1}\right\rceil}\left(\begin{array}{l}
k \\
a
\end{array}\right) \cdot\left(\begin{array}{c}
2 k+1+m \\
b
\end{array}\right) .
$$

ProOF. We can easily check that $v$ consists of $n=3 k+3+m$ players having 4 different non-negative weights. For the ease of notation we denote coalition types as a 4 -tuple $\left(c_{1}, c_{2}, c_{3}, c_{4}\right)$ meaning a coalition having $0 \leq c_{1} \leq 1$ players of weight $t+1+m(k+1), 0 \leq c_{2} \leq 1$ players of weight $t+m(k+1), 0 \leq c_{3} \leq k$ players of weight $2 k+3$, and $0 \leq c_{4} \leq 2 k+1+m$ players of weight $k+1$. Due to symmetry it suffices to know the counts $c_{1}, \ldots, c_{4}$ instead of the precise coalitions.

Note that the sum of weights of the players of weight $2 k+3$ is given by $t-1$. The sum of weights of players of weight $k+1$ is given by $t+m(k+1)$. Next we show that the players of the 4 weight types are non-equivalent. Since $(1,1,0,0)$ is winning and $(1,0,1,0)$ is losing, the player of weight $t+m(k+1)$ is non-equivalent to players of weight $2 k+3$. Since $(1,0, k, 0)$ and winning and $(0,1, k, 0)$ is losing the first two players are non-equivalent. Since $(1,0, k, 0)$ and winning and $(1,0,0, k)$ is losing, also the players of weight $2 k+3$ are non-equivalent to players of weight $k+1$. Due to the decreasing sequence of weights, we have four equivalence classes of players coinciding with the sets of players that have an equal weight.

A coalition type $(1,1, a, b)$ corresponds to swing coalitions for player 1 if and only if $a(2 k+3)+b(k+1) \leq t-1$. Similarly, a coalition type $(1,0, a, b)$ corresponds to swing coalitions for player 1 if and only if $a(2 k+3)+b(k+1) \geq t-1$. So we are interest in the number of cases where $a(2 k+3)+b(k+1)=t-1$. Considering this equation modulo $k+1$ yields $a \equiv-1(\bmod k)+1$, so that $a=k, b=0$ is the unique solution. Thus we have $\mathrm{Bz}_{1}^{r}(v)=2^{n-2}+1$. 
A coalition type $(1,1, a, b)$ corresponds to swing coalitions for player 2 if and only if $a(2 k+3)+b(k+1) \leq t-2$ and a coalition type $(0,1, a, b)$ corresponds to swing coalitions for player 2 if and only if $a(2 k+3)+b(k+1) \geq t$. Thus we have $\mathrm{Bz}_{1}^{r}(v)=2^{n-2}-1$.

The coalition $\{1,2\}$ is a minimal winning coalition in any case. We can easily check that it is shift-minimal winning if and only if $k>1$. Since the weight of $(1,0, k, 0)$ exactly equals the quota, the corresponding unique coalition is shiftminimal in any case. Now assume that a coalition $S$ of type $(1,0, a, b)$ is shiftminimal winning. Since $S$ has to be winning, we have $a(2 k+3)+b(k+1) \geq t-1$. Since $(0,1, a, b)$ has to be losing, we have $a(2 k+3)+b(k+1)=t-1$. As mentioned before, the unique solution of this equation is given by $a=k$ and $b=0$. Thus, we have $\mathrm{S}_{1}^{r}(v)=1$ for $k=1$ and $\mathrm{S}_{1}^{r}(v)=2$ for $k>1$.

Every minimal winning coalition besides $\{1,2\}$ containing player 2 has to be of type $(0,1, a, b)$. For any $0 \leq a \leq k$ the unique value for $b$ is given by $b(a)=$ $\left\lceil\frac{t-a(2 k+3)}{k+1}\right]$, where $1 \leq b \leq 2 k+1$. Since $2 k+3>2 \cdot(k+1)$ we have $b(a)+1<$ $b(a-1)$, i.e., the corresponding coalitions are shift-minimal winning. Counting the number of coalitions of type $(0,1, a, b(a))$ gives

$$
\mathrm{S}_{2}^{r}(v)=-1+\mathrm{S}_{1}^{r}(v)+\sum_{a=0}^{k} \sum_{b=\left\lceil\frac{t-a(2 k+3)}{k+1}\right\rceil}\left(\begin{array}{l}
k \\
a
\end{array}\right) \cdot\left(\begin{array}{c}
2 k+1+m \\
b
\end{array}\right) .
$$

Of course one may speculate whether the stated construction is tight in general. At the very least we can conclude that $c_{\mathcal{P}}(n, \mathfrak{W})$ tends at least exponentially to 1 , i.e., there are constants $d_{1}>0, d_{2}>1$ with $c_{\mathcal{P}}(n, \mathfrak{W}) \geq 1-d_{1} \cdot d_{2}^{-n}$, for $\mathcal{P}=\left(\mathrm{Bz}^{r}, \mathrm{~S}^{r}\right)$. Conjecture 1 on the other hand would imply only a linear convergence rate. So, in some sense, the Shift index is even less locally monotonic than the Public Good index.

Next we go on and consider restrictions of the class of weighted games.

Lemma 11. For $\mathcal{P}=\left(\mathrm{Bz}^{r}, \mathrm{PGI}^{r}\right)$ and $n \geq 3$ we have $c_{\mathcal{P}}\left(n, \mathfrak{W}^{p}\right) \geq \max \left(0, \frac{n-4}{n-2}\right)$.

ProOF. Since $c_{\mathcal{P}}(n, \mathfrak{W}) \geq 0$ by definition, it suffices to consider weighted games with $n \geq 5$ players. We consider the weighted game $v=[2 n-3 ; n-1, n-2, n-$ $2,1, \ldots, 1]$ with $n-3$ players of weight 1 , two players of weight $n-2>1$ and one player of weight $n-1$. Since the sum of voting weights is $4 n-8<2 \cdot(2 n-3)$, the game is proper. The minimal winning coalitions are given by $\{1,2\},\{1,3\}$, and $\{2,3, i\}$ for all $4 \leq i \leq n$. Thus, we have $\operatorname{PGI}^{r}(v)=(2, n-2, n-2,1, \ldots, 1)$. The swing coalitions for player 1 are given by $\{1,2,3\}$ and all coalitions of the form $\{1, i\} \cup S$, where $i \in\{2,3\}$ and $S \subseteq\{4, \ldots, n\}$. The swing coalitions for player 2 are given by $\{1,2\}$ and all coalitions of the form $\{2,3\} \cup S$, where $\emptyset \neq S \subseteq\{4, \ldots, n\}$. The unique swing coalition for a player $i \geq 4$ is given by $\{2,3, i\}$. Thus, we have $\mathrm{Bz}^{r}(v)=\left(2^{n-2}+1,2^{n-2}-1,2^{n-2}-1,1, \ldots, 1\right)$.

For players 1, 2 and game $v$ Inequality $(2)$ is equivalent to $2 \alpha_{1} \geq \alpha_{1} \cdot \mathrm{Bz}_{2}^{r}(v)+$ $(n-4) \alpha_{2}$. Inserting $\alpha_{1}+\alpha_{2}=1$ yields $\alpha_{1} \geq \frac{n-4}{n-2}$.

Corollary 6. For $\mathcal{P}=\left(\mathrm{Bz}^{r}, \mathrm{~S}^{r}\right)$ and $n \geq 3$ we have $c_{\mathcal{P}}\left(n, \mathfrak{W}^{p}\right) \geq \max \left(0, \frac{n-4}{n-2}\right)$.

Using the ILP approach we can verify that Lemma 11 is tight for $n \leq 10$ and Corollary 6 is tight for $n \leq 6$. 
Lemma 12. Let $\mathcal{P}=\left(\mathrm{Bz}^{r}, \mathrm{~S}^{r}\right)$.

(1) For $n=7$ we have $c_{\mathcal{P}}\left(n, \mathfrak{W}^{p}\right)=\frac{5}{7} \approx 0.71429$.

(2) For $n=8$ we have $c_{\mathcal{P}}\left(n, \mathfrak{W}^{p}\right)=\frac{11}{13} \approx 0.84615$.

(3) For $n=9$ we have $c_{\mathcal{P}}\left(n, \mathfrak{W}^{p}\right)=\frac{12}{13} \approx 0.92308$.

(4) For $n=10$ we have $c_{\mathcal{P}}\left(n, \mathfrak{W}^{p}\right)=\frac{21}{22} \approx 0.95455$.

(5) For $n=11$ we have $c_{\mathcal{P}}\left(n, \mathfrak{W}^{p}\right)=\frac{83}{85} \approx 0.97647$.

Proof. For the lower bounds we state an explicit weighted game and the Banzhaf and the Shift score for the first two players:

(1) $[21 ; 11,10,5,5,3,3,3], \mathrm{Bz}^{r}=(33,31, \ldots), \mathrm{S}^{r}=(2,7, \ldots)$;

(2) $[25 ; 13,12,5,5,3,3,3,3], \mathrm{Bz}^{r}=(65,63, \ldots), \mathrm{S}^{r}=(2,13, \ldots)$;

(3) $[31 ; 16,15,7,7,3,3,3,3,3], \mathrm{Bz}^{r}=(129,127, \ldots), \mathrm{S}^{r}=(2,26, \ldots)$;

(4) $[39 ; 12,11,9,9,9,5,5,5,5,5], \mathrm{Bz}^{r}=(194,192, \ldots), \mathrm{S}^{r}=(1,45, \ldots)$;

(5) $[32 ; 8,7,7,7,7,4,4,4,4,4,4], \mathrm{Bz}^{r}=(324,322, \ldots), \mathrm{S}^{r}=(1,84, \ldots)$.

For the upper bounds we have applied the ILP approach.

Since the parametric example from Lemma 8 and the examples from Lemma 9 and Lemma 10 have the property that the sum of weights meets or exceeds twice the quota, the respective games are all strong. Thus, the same results are valid if we restrict the class of weighted games to weighted strong games. Clearly we also conjecture $c_{\mathcal{P}}\left(n, \mathfrak{W}^{s}\right)=\max \left(0, \frac{n-3}{n-1}\right)$ for all $n \geq 2$, where $\mathcal{P}=\left(\mathrm{Bz}^{r}, \mathrm{PGI}^{r}\right)$, which is a weakening of Conjecture 1 .

Since the parametric example from Lemma 11 and the examples from Lemma 12 are not strong, the cost of local monotonicity may decrease for constant sum weighted games compared to proper weighted games.

If the class of weighted games is restricted to constant-sum games, then the nonmonotonicity is generally reduced, i.e. a lower contribution of the Banzhaf score is sufficient to guarantee monotonicity of the power index obtained as a convex combination.

Lemma 13. For $\mathcal{P}=\left(\mathrm{Bz}^{r}, \mathrm{PGI}^{r}\right)$ and $n \geq 2$ we have $c_{\mathcal{P}}\left(n, \mathfrak{W}^{c}\right) \geq \max \left(0, \frac{n-5}{n-1}\right)$.

Proof. Since $c_{\mathcal{P}}(n, \mathfrak{W}) \geq 0$ by definition, it suffices to consider weighted games with $n \geq 6$ players. For the weighted game $v=[2 n-5 ; n-2, n-3, n-3,1 \ldots, 1]$, with $n-3$ players of weight 1 , the minimal winning coalitions are given by $\{1,2\}$, $\{1,3\},\{1,4,5, \ldots, n\}$, and $\{2,3, i\}$, where $4 \leq i \leq n$. Thus, we have $\operatorname{PGI}^{\mathrm{S}}{ }_{1}(v)=3$ and $\mathrm{PGI}_{2}{ }_{2}(v)=n-2$. For player 1 the swing coalitions are given by $\{1,2,3\}$, $\{1,4,5, \ldots, n\}$, and $\{1,2\} \cup S,\{1,3\} \cup S$, where $S \subseteq\{4,5, \ldots, n\}$. For player 2 the swing coalitions are given by $\{1,2\} \cup U$, where $U \subseteq\{4,5, \ldots, n\}$ with $|U|<n-3$, and $\{2,3\} \cup V$, where $\emptyset \neq V \subseteq\{4,5, \ldots, n\}$. Thus, we have $\mathrm{BZ}_{1}^{\mathrm{S}}(v)=2^{n-2}+2$ and $\mathrm{BZ}_{2}(v)=2^{n-2}-2$.

For players 1,2 and game $v$ Inequality (2) is equivalent to $4 \alpha_{1} \geq \alpha_{1} \cdot \mathrm{Bz}_{2}^{r}(v)+$ $(n-5) \alpha_{2}$. Inserting $\alpha_{1}+\alpha_{2}=1$ yields $\alpha_{1} \geq \frac{n-5}{n-1}$.

Corollary 7. For $\mathcal{P}=\left(\mathrm{Bz}^{r}, \mathrm{~S}^{r}\right)$ and $n \geq 2$ we have $c_{\mathcal{P}}\left(n, \mathfrak{W}^{c}\right) \geq \max \left(0, \frac{n-5}{n-1}\right)$.

\section{Corollary 8.}

$$
\lim _{n \rightarrow \infty} c_{\left(\mathrm{Bz}^{r}, \mathrm{PGI}^{r}\right)}\left(n, \mathfrak{W}^{c}\right)=\lim _{n \rightarrow \infty} c_{\left(\mathrm{Bz}^{r}, \mathrm{~S}^{r}\right)}\left(n, \mathfrak{W}^{c}\right)=\lim _{n \rightarrow \infty} c_{\left(\mathrm{Bz}^{r}, \mathrm{PGI}^{r}, \mathrm{~S}^{r}\right)}\left(n, \mathfrak{W}^{c}\right)=1
$$


Using the ILP approach we can verify that Lemma 13 is tight for $n \leq 11$ and Corollary 7 is tight for $n \leq 7$.

Lemma 14. Let $\mathcal{P}=\left(\mathrm{Bz}^{r}, \mathrm{~S}^{r}\right)$.

(1) For $n=8$ we have $c_{\mathcal{P}}\left(n, \mathfrak{W}^{c}\right)=\frac{2}{3} \approx 0.66667$.

(2) For $n=9$ we have $c_{\mathcal{P}}\left(n, \mathfrak{W}^{c}\right)=\frac{23}{27} \approx 0.85185$.

(3) For $n=10$ we have $c_{\mathcal{P}}\left(n, \mathfrak{W}^{c}\right)=\frac{43}{47} \approx 0.91489$.

(4) For $n=11$ we have $c_{\mathcal{P}}\left(n, \mathfrak{W}^{c}\right)=\frac{75}{79} \approx 0.94937$.

Proof. For the lower bounds we state an explicit weighted game and the Banzhaf and the Shift score for the first two players:

(1) $[17 ; 9,8,5,3,2,2,2,2], \mathrm{Bz}^{r}=(66,62, \ldots), \mathrm{S}^{r}=(3,11, \ldots)$;

(2) $[21 ; 11,10,5,5,2,2,2,2,2], \mathrm{Bz}^{r}=(130,126, \ldots), \mathrm{S}^{r}=(3,26, \ldots)$;

(3) $[21 ; 6,5,5,5,5,3,3,3,3,3], \mathrm{Bz}^{r}=(170,166, \ldots), \mathrm{S}^{r}=(5,48, \ldots)$;

(4) $[22 ; 8,7,7,7,2,2,2,2,2,2,2], \mathrm{Bz}^{r}=(386,382, \ldots), \mathrm{S}^{r}=(4,79, \ldots)$.

For the upper bounds we have applied the ILP approach.

Similar results can be obtained for $\mathrm{Jo}^{r}, \mathrm{DP}^{r}$, and $\mathrm{SDP}^{r}$.

Lemma 15. Let $\mathcal{P}=\left(\mathrm{Jo}^{r}, \mathrm{DP}^{r}\right)$.

(1) For $n \leq 4$ we have $c_{\mathcal{P}}(n, \mathfrak{W})=0$.

(2) For $n=5$ we have $c_{\mathcal{P}}(n, \mathfrak{W})=\frac{1}{8}=0.125$.

(3) For $n=6$ we have $c_{\mathcal{P}}(n, \mathfrak{W})=\frac{1}{4}=0.25$.

(4) For $n=7$ we have $c_{\mathcal{P}}(n, \mathfrak{W})=\frac{1}{3} \approx 0.33333$.

(5) For $n=8$ we have $c_{\mathcal{P}}(n, \mathfrak{W})=\frac{2}{5}=0.4$.

(6) For $n=9$ we have $c_{\mathcal{P}}(n, \mathfrak{W}) \geq \frac{11}{25}=0.44$.

(7) For $n=10$ we have $c_{\mathcal{P}}(n, \mathfrak{W}) \geq \frac{1}{2}=0.5$.

Proof. For the lower bounds we state an explicit weighted game, the Johnston and the Deegan-Packel score, where we highlight the values of the critical players:

(2) $[3 ; 3,2,2,1,1], \mathrm{Jo}^{r}=\left(\mathbf{6}, \frac{\mathbf{5}}{\mathbf{2}}, \frac{5}{2}, 1,1\right), \mathrm{SDP}^{r}=\left(\mathbf{1}, \frac{\mathbf{3}}{\mathbf{2}}, \frac{3}{2}, 1,1\right)$;

(3) $[8 ; 4,4,3,1,1,1], \mathrm{Jo}^{r}=\left(\frac{15}{2}, \frac{\mathbf{1 5}}{\mathbf{2}}, \mathbf{6}, \frac{2}{3}, \frac{2}{3}, \frac{2}{3}\right), \mathrm{SDP}^{r}=\left(\frac{3}{2}, \frac{\mathbf{3}}{\mathbf{2}}, \mathbf{2}, \frac{2}{3}, \frac{2}{3}, \frac{2}{3}\right)$;

(4) $[9 ; 5,4,3,2,2,2,2], \mathrm{Jo}^{r}=\left(\frac{70}{3}, \frac{\mathbf{2 8}}{\mathbf{3}}, \frac{\mathbf{2 3}}{\mathbf{3}}, \frac{19}{6}, \frac{19}{6}, \frac{19}{6}, \frac{19}{6}\right)$, $\mathrm{SDP}^{r}=\left(\frac{23}{6}, \frac{17}{6}, \frac{11}{3}, \frac{19}{6}, \frac{19}{6}, \frac{19}{6}, \frac{19}{6}\right) ;$

(5) $[12 ; 4,4,4,3,3,3,3,3], \mathrm{Jo}^{r}=\left(\frac{19}{2}, \frac{19}{2}, \frac{19}{2}, \frac{17}{2}, \frac{17}{2}, \frac{17}{2}, \frac{17}{2}, \frac{17}{2}\right)$, $\mathrm{SDP}^{r}=\left(\frac{47}{6}, \frac{47}{6}, \frac{47}{6}, \frac{17}{2}, \frac{17}{2}, \frac{17}{2}, \frac{17}{2}, \frac{17}{2}\right) ;$

(6) $[20 ; 5,5,5,5,4,4,4,4,4], \mathrm{Jo}^{r}=\left(\frac{19}{2}, \ldots, \frac{\mathbf{1 9}}{\mathbf{2}}, \frac{\mathbf{6 9}}{\mathbf{5}}, \ldots, \frac{69}{5}\right)$, $\mathrm{SDP}^{r}=\left(\frac{53}{4}, \ldots, \frac{53}{4}, \frac{69}{5}, \ldots, \frac{69}{5}\right)$

(7) $[20 ; 5,5,5,5,4,4,4,4,4,4], \mathrm{Jo}^{r}=\left(\frac{103}{4}, \ldots, \frac{\mathbf{1 0 3}}{\mathbf{4}}, \mathbf{2 5}, \ldots, 25\right)$, $\mathrm{SDP}^{r}=\left(\frac{97}{4}, \ldots, \frac{97}{4}, \mathbf{2 5}, \ldots, 25\right)$

For the upper bounds we have applied the ILP approach.

For $\mathcal{P}=\left(\mathrm{Jo}^{r}, \mathrm{DP}^{r}\right)$ the cost of local monotonicity seems to be increasing rather slowly. Given the numerical data from Lemma 15 it is not clear at all whether $c_{\mathcal{P}}(n, \mathfrak{W})$ tends to 1 as $n$ tends to infinity. To this end we consider the following construction for a odd number of players:

Lemma 16. For $k \geq 1$ and $v=[k(k+1), \overbrace{k+1, \ldots, k+1}^{k \text { times }}, \overbrace{k, \ldots, k}^{k+1}]$ we have $\mathrm{Jo}_{i}^{r}(v)=c(k)$ for all $1 \leq i \leq k, \mathrm{Jo}_{i}^{r}(v)=d(k)$ for all $k+1 \leq 2 k+1, \mathrm{DP}_{i}^{r}(v)=$ 
$c(k)-\frac{k+1}{k}$ for all $1 \leq i \leq k$, and $\mathrm{DP}_{i}^{r}(v)=d(k)$ for all $k+1 \leq 2 k+1$, where

$$
c(k)=\frac{k+2}{k}+\frac{1}{k+1} \sum_{i=1}^{k-1}\left(\begin{array}{c}
k-1 \\
i-1
\end{array}\right) \cdot\left(\begin{array}{c}
k+1 \\
i
\end{array}\right)
$$

and

$$
d(k)=\frac{1}{k+1}+\frac{1}{k+1} \cdot \sum_{i=1}^{k-1}\left(\begin{array}{l}
k \\
i
\end{array}\right)^{2} .
$$

Proof. We can easily check that $v$ consists of $n=2 k+1$ players having two different weights. As in the proof of Lemma 10 we use a 2-tuple $\left(c_{1}, c_{2}\right)$ to describe the type of a coalition. Since the coalitions of type $(k, 0)$ are winning but the coalitions of type $(0, k)$ are losing, no player of weight $k+1$ is equivalent to a player of weight $k$.

In coalitions of type $(k, 0)$ or type $(k, 1)$ all players of weight $k+1$ are swing players, while the players of weight $k$ are not swing players. The other types of coalitions which contain a least one swing player are given by $(i, k+1-i)$, where $0 \leq i \leq k-1$. In these cases all involved $k+1$ players are swings. Counting the number of cases, where player 1 is contained, for each of the mentioned coalition types gives

$$
\begin{aligned}
\mathrm{Jo}_{1}^{r}(v)= & \left(\begin{array}{l}
k-1 \\
k-1
\end{array}\right) \cdot\left(\begin{array}{c}
k+1 \\
0
\end{array}\right) \cdot \frac{1}{k}+\left(\begin{array}{c}
k-1 \\
k-1
\end{array}\right) \cdot\left(\begin{array}{c}
k+1 \\
1
\end{array}\right) \cdot \frac{1}{k} \\
& +\frac{1}{k+1} \cdot \sum_{i=1}^{k-1}\left(\begin{array}{c}
k-1 \\
i-1
\end{array}\right) \cdot\left(\begin{array}{c}
k+1 \\
i
\end{array}\right) \\
= & c(k)
\end{aligned}
$$

Counting the number of cases, where player $n$ is contained, for each of the mentioned coalition types gives

$$
\mathrm{Jo}_{n}^{r}(v)=\frac{1}{k+1}+\frac{1}{k+1} \cdot \sum_{i=1}^{k-1}\left(\begin{array}{c}
k \\
i
\end{array}\right) \cdot\left(\begin{array}{c}
k \\
i
\end{array}\right)=d(k) .
$$

All coalition types except $(k, 1)$ correspond to minimal winning coalitions. Thus we have $\mathrm{DP}_{1}^{r}(v)=\mathrm{Jo}_{1}^{r}(v)-\left(\begin{array}{c}k-1 \\ k-1\end{array}\right) \cdot\left(\begin{array}{c}k+1 \\ 1\end{array}\right) \cdot \frac{1}{k}=c(k)-\frac{k+1}{k}$ and $\mathrm{DP}_{n}^{r}(v)=\mathrm{Jo}_{n}^{r}(v)=d(k)$. The values for the remaining players follow from symmetry.

Corollary 9. For $\mathcal{P}=\left(\mathrm{Jo}^{r}, \mathrm{DP}^{r}\right)$ and $n \geq 1$ we have $c_{\mathcal{P}}(n, \mathfrak{W}) \geq 1-\frac{2(3 n-2)}{n^{2}} \geq$ $1-\frac{6}{n}$.

Proof. For the weighted game from Lemma 16 Inequality 22 yields $c_{\mathcal{P}}(2 k+$ $1, \mathfrak{W}) \geq 1-\frac{k}{k+1} \cdot(c(k)-d(k))$. Since $\sum_{i=1}^{k-1}\left(\begin{array}{c}k-1 \\ i-1\end{array}\right) \cdot\left(\begin{array}{c}k+1 \\ i\end{array}\right)-\sum_{i=1}^{k-1}\left(\begin{array}{c}k \\ i\end{array}\right)^{2}=-(k-1)$, we have $c(k)-d(k)=\frac{3 k+2}{k(k+1)}$. Thus $c_{\mathcal{P}}(2 k+1, \mathfrak{W}) \geq 1-\frac{3 k+2}{(k+1)^{2}}$. Since $c_{\mathcal{P}}(n, \mathfrak{W}) \geq$ $c_{\mathcal{P}}(n-1, \mathfrak{W})$ we can choose $k=\left\lceil\frac{n-2}{2}\right\rceil$ and obtain the stated lower bounds.

Corollary 10. For $\mathcal{P}=\left(\mathrm{Jo}^{r}, \mathrm{DP}^{r}\right)$ we have $\lim _{n \rightarrow \infty} c_{\mathcal{P}}(n, \mathfrak{W})=1$.

Lemma 17. Let $\mathcal{P}=\left(\mathrm{Jo}^{r}, \mathrm{SDP}^{r}\right)$.

(1) For $n \leq 4$ we have $c_{\mathcal{P}}(n, \mathfrak{W})=0$.

(2) For $n=5$ we have $c_{\mathcal{P}}(n, \mathfrak{W})=\frac{1}{3} \approx 0.33333$.

(3) For $n=6$ we have $c_{\mathcal{P}}(n, \mathfrak{W})=\frac{3}{5}=0.6$. 
(4) For $n=7$ we have $c_{\mathcal{P}}(n, \mathfrak{W})=\frac{7}{9} \approx 0.77778$.

(5) For $n=8$ we have $c_{\mathcal{P}}(n, \mathfrak{W})=\frac{47}{53} \approx 0.88679$.

(6) For $n=9$ we have $c_{\mathcal{P}}(n, \mathfrak{W}) \geq \frac{29}{31} \approx 0.93548$.

Proof. For the lower bounds we state an explicit weighted game and the Johnston and the Shift Deegan-Packel score for the last two players:

(2) $[4 ; 3,3,2,2,1], \mathrm{Jo}^{r}=(\ldots, 2,1), \mathrm{SDP}^{r}=\left(\ldots, \frac{1}{2}, 1\right)$;

(3) $[8 ; 4,4,3,3,2,1], \mathrm{Jo}^{r}=\left(\ldots, 2, \frac{4}{3}\right), \mathrm{SDP}^{r}=\left(\ldots, \frac{1}{3}, \frac{4}{3}\right)$;

(4) $[8 ; 5,5,2,2,2,2,1], \mathrm{Jo}^{r}=\left(\ldots, \frac{19}{6}, \frac{8}{3}\right), \mathrm{S}^{r}=\left(\ldots, \frac{11}{12}, \frac{8}{3}\right)$;

(5) $[15 ; 7,7,3,3,3,3,3,2], \mathrm{Jo}^{r}=\left(\ldots, \frac{86}{15}, \frac{16}{3}\right), \mathrm{SDP}^{r}=\left(\ldots, \frac{11}{5}, \frac{16}{3}\right)$;

(6) $[12 ; 7,7,2,2,2,2,2,2,1], \mathrm{Jo}^{r}=\left(\ldots, \frac{47}{6}, \frac{15}{2}\right), \mathrm{SDP}^{r}=\left(\ldots, \frac{8}{3}, \frac{15}{2}\right)$.

For the upper bounds we have applied the ILP approach.

Quite obviously the cost of local monotonicity for $\mathcal{P}=\left(\mathrm{Jo}^{r}, \mathrm{DP}^{r}\right)$ seems to converge to 1 as $n$ increases. An appropriate lower bound can be concluded from the parametric example $v=[2(n-3) ; n-2, n-2, \underbrace{2, \ldots, 2}_{n-3 \text { times }}, 1]$, where $n \geq 5$, by considering the last two players. We remark that the exact value for $n=7$ and the lower bound for $n=9$ is attained for this parametric family.

\section{Determining the polyhedron $\mathbb{P}_{\text {LM }}^{\mathcal{P}}(n, \mathfrak{W})$ For Convex Combinations of THREE POWER INDICES.}

In the previous section we have computationally determined the cost of local monotonicity for several sets of two or three power indices on subclasses of weighted games. Now we want to gain even more information: Given a collection $\mathcal{P}$ of $r \geq 2$ power indices, for which $\alpha \in \mathbb{S}^{r}$ does $P^{\alpha, \mathcal{P}}$ satisfy local monotonicity? In Section 4 we have obtained the result that the respective set $\mathbb{P}_{\mathrm{LM}}^{\mathcal{P}}(n, \mathfrak{G})$ is a polyhedron. As already discussed, each game $v \in \mathfrak{G}$ gives a valid inequality for $\mathbb{P}_{\mathrm{LM}}^{\mathcal{P}}(n, \mathfrak{G})$. Using the ILP approach from Section 5 we can check whether a given point $\alpha \in \mathbb{S}^{r}$ is contained in $\mathbb{P}_{\mathrm{LM}}^{\mathcal{P}}(n, \mathfrak{G})$. In the case where $\alpha$ is not contained in $\mathbb{P}_{\mathrm{LM}}^{\mathcal{P}}(n, \mathfrak{G})$, we obtain a game $v \in \mathfrak{G}$ verifying this fact. So either we can verify vertices of our polyhedron or compute additional non-redundant valid inequalities. So, instead of looping over all games in $\mathfrak{G}$, we can use the following algorithm to determine $\mathbb{P}_{\mathrm{LM}}^{\mathcal{P}}(n, \mathfrak{G})$ :

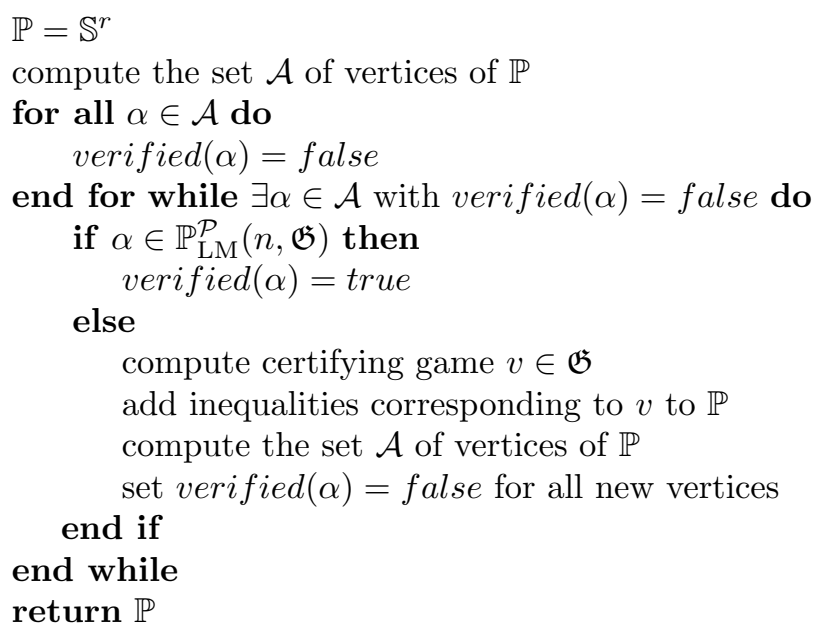


We remark that we may also include the information that $(1,0, \ldots, 0) \in \mathbb{P}_{\text {LM }}^{\mathcal{P}}(n, \mathfrak{G})$ in any case, i.e., one of the $r$ vertices of $\mathbb{S}^{r}$ can be set to be verified. If already determined, the $r-1$ examples for the cost of local monotonicity for $\mathcal{P}^{\prime}=\left\{P^{1}, P^{i}\right\}$ can be used to replace the initialization of $\mathbb{P}$, i.e., setting $\mathbb{P}=\operatorname{conv}\left(e_{1}, p_{2}, \ldots, p_{r}\right)$, where $e_{i}$ is the $i$ th unit vector and $p_{i}=e_{1} \cdot c_{\left\{P^{1}, P^{i}\right\}}(n, \mathfrak{G})+e_{i} \cdot\left(1-c_{\left\{P^{1}, P^{i}\right\}}(n, \mathfrak{G})\right)$.

Exemplarily, we have performed the computations for $\mathfrak{G}=\mathfrak{W}, \mathcal{P}=\left\{\mathrm{Bz}^{r}, \mathrm{PGI}^{r}, \mathrm{~S}^{r}\right\}$, and $n \leq 9$.

Lemma 18. For $\mathcal{P}=\left\{\mathrm{Bz}^{r}, \mathrm{PGI}^{r}, \mathrm{~S}^{r}\right\}$ we have

(1) $\mathbb{P}_{L M}^{\mathcal{P}}(n, \mathfrak{W})=\mathbb{S}^{3}=\operatorname{conv}\{(1,0,0),(0,1,0),(0,0,1)\}$ for $n \leq 3$;

(2) $\mathbb{P}_{L M}^{\mathcal{P}}(4, \mathfrak{W})=\operatorname{conv}\left\{(1,0,0),\left(\frac{1}{3}, \frac{2}{3}, 0\right),\left(\frac{1}{3}, 0, \frac{2}{3}\right)\right\}$;

(3) $\mathbb{P}_{L M}^{\mathcal{P}}(5, \mathfrak{W})=\operatorname{conv}\left\{(1,0,0),\left(\frac{1}{2}, \frac{1}{2}, 0\right),\left(\frac{1}{2}, 0, \frac{1}{2}\right)\right\}$;

(4) $\mathbb{P}_{L M}^{\mathcal{P}}(6, \mathfrak{W})=\operatorname{conv}\left\{(1,0,0),\left(\frac{3}{5}, \frac{2}{5}, 0\right),\left(\frac{3}{5}, 0, \frac{2}{5}\right)\right\}$;

(5) $\mathbb{P}_{L M}^{\mathcal{P}}(7, \mathfrak{W})=\operatorname{conv}\left\{(1,0,0),\left(\frac{2}{3}, \frac{1}{3}, 0\right),\left(\frac{7}{9}, 0, \frac{2}{9}\right),\left(\frac{2}{3}, \frac{1}{4}, \frac{1}{12}\right)\right\}$;

(6) $\mathbb{P}_{L M}^{\mathcal{P}}(8, \mathfrak{W})=\operatorname{conv}\left\{(1,0,0),\left(\frac{5}{7}, \frac{2}{7}, 0\right),\left(\frac{7}{8}, 0, \frac{1}{8}\right),\left(\frac{5}{7}, \frac{9}{35}, \frac{1}{35}\right)\right\}$;

(7) $\mathbb{P}_{L M}^{\mathcal{P}}(9, \mathfrak{W})=\operatorname{conv}\left\{(1,0,0),\left(\frac{3}{4}, \frac{1}{4}, 0\right),\left(\frac{25}{27}, 0, \frac{2}{27}\right),\left(\frac{3}{4}, \frac{19}{80}, \frac{1}{80}\right)\right\}$.

In Figure 2 we have exemplarily drawn $\mathbb{P}_{\mathrm{LM}}^{\left\{\mathrm{Bz}^{r}, \mathrm{PGI}^{r}, \mathrm{~S}^{r}\right\}}(7, \mathfrak{W})$, which complements the region drawn in Figure 1. In order to illustrate the proposed algorithm we consider the case $n=9$ as an example. For $\left\{\mathrm{Bz}^{r}, \mathrm{PGI}^{r}\right\}$ the cost of local monotonicity is given by $\frac{3}{4}$ and e.g. attained at the game $v_{1}=[2 ; 2,1,1,1,1,1,1,1,1]$. We have $\operatorname{Bz}^{r}\left(v_{1}\right)=(9,7, \ldots), \operatorname{PGI}^{r}\left(v_{1}\right)=(1,7, \ldots)$, and $\mathrm{S}^{r}\left(v_{1}\right)=(1,7, \ldots)$. For $\left\{\mathrm{Bz}^{r}, \mathrm{~S}^{r}\right\}$ the cost of local monotonicity is given by $\frac{25}{27}$ and e.g. attained at the game $v_{2}=[30 ; 16,15,7,7,3,3,3,3,3]$. We have $\operatorname{Bz}^{r}\left(v_{2}\right)=(129,127, \ldots)$, $\operatorname{PGI}^{r}\left(v_{2}\right)=(23,27, \ldots)$, and $\mathrm{S}^{r}\left(v_{2}\right)=(2,27, \ldots)$. The hyperplane corresponding to $v_{1}$ is given by $2 \alpha_{1}-6 \alpha_{2}-6 \alpha_{3}=0$ and the hyperplane corresponding to $v_{2}$ is given by $2 \alpha_{1}-4 \alpha_{2}-25 \alpha_{3}$. Together with $\alpha_{1}+\alpha_{2}+\alpha_{3}=1$ we obtain the new vertex $\alpha^{\prime}=\left(\frac{3}{4}, \frac{19}{84}, \frac{1}{42}\right)$. By using the ILP approach we can compute that $\alpha^{\prime}$ does not lead to a locally monotonic power index and obtain the game $v_{3}=$ $[18 ; 13,12,5,2,2,2,2,2,2]$ with $\mathrm{Bz}^{r}\left(v_{3}\right)=(129,127, \ldots), \operatorname{PGI}^{r}\left(v_{3}\right)=(22,27, \ldots)$, and $\mathrm{S}^{r}\left(v_{3}\right)=(1,26, \ldots)$. For this game the corresponding hyperplane is given by $2 \alpha_{1}-5 \alpha_{2}-25 \alpha_{3}$. Again there arises exactly one new vertex $-\alpha^{\prime \prime}=\left(\frac{3}{4}, \frac{19}{80}, \frac{1}{80}\right)$. By using the ILP approach we can compute that $\alpha^{\prime \prime}$ is contained in $\mathbb{P}_{\mathrm{LM}}^{\mathcal{P}}(9, \mathfrak{W})$, so that the determination of the polyhedron is completed.

We remark that $\alpha^{\prime \prime}$ does also attain the cost of local monotonicity for $\left\{\mathrm{Bz}^{r}, \mathrm{~S}^{r}\right\}$. So if we had started with the games $v_{1}$ and $v_{3}$ instead of $v_{1}$ and $v_{2}$, our algorithm would have needed one iteration less.

\section{Conclusion}

We have introduced the concept of considering convex combinations of power indices. Several of the main properties of power indices are preserved by convexity, i.e., given a collection $\mathcal{P}$ of power indices such that each power index in $\mathcal{P}$ has a certain property, then also every convex combination of the power indices in $\mathcal{P}$ has this property. Lemma 1 gives some examples of such properties being preserved by convexity. The freedom in choosing the multipliers almost arbitrarily enables us to search for power indices, which satisfy some other useful properties. As an application we study local monotonicity. It is well known that the Public Good index does not satisfy local monotonicity, while e.g. the Banzhaf index does. So, 


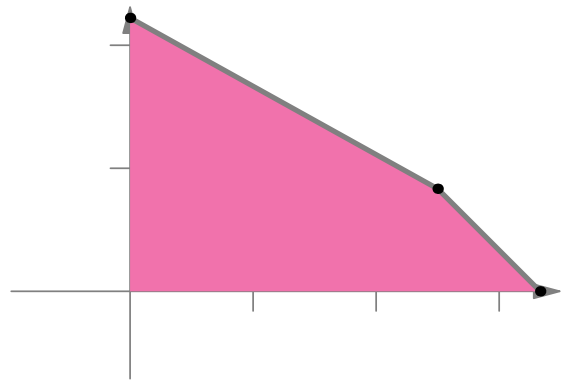

Figure 2. $\mathbb{P}_{\mathrm{LM}}^{\mathcal{P}}(7, \mathfrak{W})$ for $\mathcal{P}=\left\{\mathrm{Bz}^{r}, \mathrm{PGI}^{r}, \mathrm{~S}^{r}\right\}$.

what proportion of the Banzhaf index is necessary so that a convex combination of both indices becomes locally monotonic? The newly introduced cost of local monotonicity answers this specific question. Similar measures may of course be introduced for other properties of power indices.

It turns out that with an increasing number of players the weight of the Banzhaf index needs to tend to 1. For a finite number of players there is still some freedom to incorporate some information from the Public Good index, while maintaining the local monotonicity of the Banzhaf index.

Restricting the class of the underlying games to strong or proper games typically decreases the cost of local monotonicity, but does not change the general behavior.

The cost of local monotonicity for combinations of the Banzhaf and the Shift index is considerably higher. So, in some sense the Shift index is even less locally monotonic than the Public Good index. Going over to the so-called equal division version of the Banzhaf-, Public Good-, and the Shift index, i.e., The Johnston-, the Deegan-Packel and the Shift-Deegan Packel index, seems to generally lower the cost of local monotonicity. Nevertheless, the corresponding cost of local monotonicity approaches 1 as the number of players tends to infinity.

So, our study has shed some light on the property of local monotonicity of some power indices. The methodology of considering convex combinations of power indices in order to obtain some desirable properties is quite general and the presented theoretical and algorithmic framework may be applied in further studies.

\section{REFERENCES}

[1] E. Algaba, J.M. Bilbao, and J.R. Fernández. The distribution of power in the European Constitution. European J. Oper. Res., 176(3):1752-1766, 2007.

[2] J.M. Alonso-Meijide and J. Freixas. A new power index based on minimal winning coalitions without any surplus. Decision Support Systems, 49(1):70-76, 2010.

[3] J.M. Alonso-Meijide, J. Freixas, and X. Molinero. Computation of several power indices by generating functions. Appl. Math. Comput., 219(8):3395-3402, 2012.

[4] J.F. Banzhaf. Weighted voting doesn't work: A mathematical analysis. Rutgers Law Rev., 19:317-343, 1965.

[5] C. Bertini, J. Freixas, G. Gambarelli, and I. Stach. Comparing power indices. Int. Game Theory Rev., 15(2), 2013.

[6] J.M. Bilbao, J.R. Fernández, N. Jiménez, and J.J. López. Voting power in the European Union enlargement. European J. Oper. Res., 143(1):181-196, 2002.

[7] J. Deegan Jr and E.W. Packel. A new index of power for simple n-person games. Internat. J. Game Theory, 7(2):113-123, 1978. 
[8] D.S. Felsenthal and M. Machover. Postulates and paradoxes of relative voting power - a critical re-appraisal. Theory and Decision, 38(2):195-229, 1995.

[9] D.S. Felsenthal and M. Machover. The measurement of voting power: Theory and practice, problems and paradoxes. Cheltenham: Edward Elgar. xviii, 322 p., 1998.

[10] J. Freixas and G. Gambarelli. Common internal properties among power indices. Control and Cybernetics, 26(4):591-603, 1997.

[11] J. Freixas and S. Kurz. On $\alpha$-roughly weighted games. Internat. J. Game Theory, 43(3):659692, 2014.

[12] J. Freixas, D. Marciniak, and M. Pons. On the ordinal equivalence of the Johnston, Banzhaf and Shapley power indices. European J. Oper. Res., 216(2):367-375, 2012.

[13] J. Freixas and X. Molinero. Weighted games without a unique minimal representation in integers. Optim. Methods Softw., 25:203-215, 2010.

[14] J. Freixas and M. A. Puente. Dimension of complete simple games with minimum. European J. Oper. Res., 188(2):555-568, 2008.

[15] M.J. Holler. Forming coalitions and measuring voting power. Political studies, 30(2):262-271, 1982.

[16] M.J. Holler and S. Napel. Monotonicity of power and power measures. Theory and Decision, 56(1-2):93-111, 2004.

[17] M.J. Holler and H. Nurmi. Reflections on power, voting, and voting power. In M.J. Holler and H. Nurmi, editors, Power, Voting, and Voting Power: 30 Years After, pages 1-24. Springer, 2013.

[18] M.J. Holler, R. Ono, and F. Steffen. Constrained monotonicity and the measurement of power. Theory and Decision, 50(4):383-395, 2001.

[19] M.J. Holler and E.W. Packel. Power, luck and the right index. Zeitschrift für Nationalökonomie, 43(1):21-29, 1983.

[20] J.R. Isbell. A class of simple games. Duke Math. J., 25:423-439, 1958.

[21] R.J. Johnston. On the measurement of power: Some reactions to Laver. Environment and Planning A, 10(8):907-914, 1978.

[22] S. Kaniovski and S. Kurz. The average representation - a cornucopia of power indices? submitted, page 10 pp., 2014. available at http://arxiv.org/abs/1405.0825

[23] V.M. Kartak, S. Kurz, A.V. Ripatti, and G. Scheithauer. Minimal proper non-irup instances of the one-dimensional cutting stock problem. Discrete Appl. Math., 2014. submitted.

[24] I. Krohn and P. Sudhölter. Directed and weighted majority games. Math. Methods Oper. Res., 42(2):189-216, 1995.

[25] S. Kurz. On minimum sum representations for weighted voting games. Ann. Oper. Res., 196(1):361-369, 2012.

[26] S. Kurz. On the inverse power index problem. Optimization, 61(8):989-1011, 2012.

[27] S. Kurz. The inverse problem for power distributions in committees. submitted, page 46 pp., 2014. available at http://arxiv.org/abs/1402.0988

[28] S. Kurz and S. Napel. Heuristic and exact solutions to the inverse power index problem for small voting bodies. Ann. Oper. Res., 215(1):137-163, 2014.

[29] S. Kurz and N. Tautenhahn. On Dedekind's problem for complete simple games. Internat. J. Game Theory, 42(2):411-437, 2013.

[30] S. Muroga. Threshold logic and its applications. New York etc.: Wiley-Interscience, a Division of John Wiley \& Sons, Inc. XIV, 478 p., 1971.

[31] W.H. Riker. The theory of political coalitions, volume 578. Yale University Press New Haven, 1962.

[32] M. Widgrén. Voting power in the EC decision making and the consequences of two different enlargements. European J. Oper. Res., 38(5):1153-1170, 1994.

Departament de Matemàtica Aplicada 3 i Escola Politècnica Superior d'Enginyeria de Manresa (Universitat Politècnica de Catalunya). Spain. Josep.freixas@upc.edu REsearch partially supported by "Ministerio de Economía y Competitividad proyecto MTM2012-34426/FEDER".

Department of Mathematics, University of Bayreuth, 95440 Bayreuth, Germany., TEL.: +49-921-557353, FAX: +49-921-557352, SASCHA.KURZ@UNI-BAYREUTH.DE 